\title{
Review Article \\ Pain Sensitivity and Observer Perception of Pain in Individuals with Autistic Spectrum Disorder
}

\author{
C. S. Allely \\ Institute of Health and Wellbeing, University of Glasgow, RHSC Yorkhill, Glasgow G3 8SJ, UK \\ Correspondence should be addressed to C. S. Allely; clare.allely@glasgow.ac.uk
}

Received 17 April 2013; Accepted 30 April 2013

Academic Editors: C. Gillberg and H. Minnis

Copyright (c) 2013 C. S. Allely. This is an open access article distributed under the Creative Commons Attribution License, which permits unrestricted use, distribution, and reproduction in any medium, provided the original work is properly cited.

\begin{abstract}
The peer-reviewed literature investigating the relationship between pain expression and perception of pain in individuals with ASD is sparse. The aim of the present systematic PRIMSA review was twofold: first, to see what evidence there is for the widely held belief that individuals with ASD are insensitive to pain or have a high pain threshold in the peer-reviewed literature and, second, to examine whether individuals with ASD react or express pain differently. Fifteen studies investigating pain in individuals with ASD were identified. The case studies all reported pain insensitivity in individuals with ASD. However, the majority of the ten experimental studies reviewed indicate that the idea that individuals with ASD are pain insensitive needs to be challenged. The findings also highlight the strong possibility that not all children with ASD express their physical discomfort in the same way as a neurotypical child would (i.e., cry, moan, seek comfort, etc.) which may lead caregivers and the medical profession to interpret this as pain insensitivity or incorrectly lead them to believe that the child is in no pain. These results have important implications for the assessment and management of pain in children with ASD.
\end{abstract}

\section{Introduction}

Autistic Spectrum Disorder (ASD) describes a range of conditions classified as pervasive developmental disorders (PDDs) in the Diagnostic and Statistical Manual of Mental Disorders (DSM). PDDS include autism, Asperger syndrome, pervasive developmental disorder not otherwise specified (PDD-NOS), childhood disintegrative disorder, and Rett syndrome. However, typically only the first three are considered part of the autism spectrum [1]. ASDs have an onset in early childhood and adverse, often lifelong, effects on communication, socialisation including tendencies toward restricted interests and/or repetitive behaviours [2]. Often associated with these symptoms are sensory-perceptual anomalies which occur in approximately $70 \%$ of cases [3]. To determine the prevalence of autism and related disorders, the Centers for Disease Control (CDC) conducted a study examining 8-year-old children living in 14 sites in the United States and found that 1 in 150 children are living with an ASD [4]. Despite the numerous studies attempting to clarify the pathogenesis of ASD, the causes remain uncertain [5].
Individuals with ASD may experience sensory abnormalities related to sight, hearing, touch, smell, and/or taste that include an increased sensitivity to pain. The processing of these types of incoming information might be distorted; rain might sound like gunfire, clothing might feel like sandpaper, or fingers shampooing a scalp might feel like sharp metal [6]. Such unusual responsiveness to the environment has been suggested to be partly due to stimulus overselectivity, the tendency of individuals with ASD to respond only to a very limited amount of the relevant sensory information [7]. Both hyposensitivity and hypersensitivity are exhibited in the same individual $[3,8-10]$. Stereotyped and self-injurious behaviours (SIBs) are also exhibited in significant numbers of individuals with ASD [11-15] which has been associated with apparent pain insensitivity [16]. As many as 70\% of ASD patients may show self-injurious behaviour at some point in their lives, but this is typically found in more severely affected individuals and takes on many forms including head banging, scratching, bruising, and biting [17]. However, the role of pain in relation to self-injury is unclear [18-22]. 
Numerous biochemical theories have been put forward to explain the apparent pain insensitivities in individuals with ASD. Certain repetitious activities such as rocking, arm flapping, or pacing produce an increase (or build up) of the level of released endorphins which can lead to a reduction of the sensation of pain, which may explain why children with an ASD who have physical accidents report feeling less pain when the accidents take place later in the day [23]. A number of researchers have also suggested that excessive brain opioid activity could explain the apparent pain insensitivity of ASD and contribute to or even determine the pathogenesis of ASD [24-30]. The opioid hypothesis for ASD postulates that this "hyperfunction of the endogenous opioid system" may actually explain some, if not all, of the symptoms associated with ASD including (1) reduced socialisation (and aloofness), (2) reduced clinging in animals, (3) diminished crying, (4) repetitive stereotyped behaviours, (5) promotion of convulsive activity, (6) insensitivity to pain, (7) episodes of motor hyperactivity alternating with hypoactivity, and (8) affective lability [27].

To date, relatively little research on the sensitivity to painful stimuli, or the expression of pain, in infants, children or adults with ASD has been conducted. Accurate pain assessment, in order to provide appropriate and timely care, can be a challenging task especially in children with ASD [31]. However, pain assessment strategies for children with ASD are poorly understood [32] and relatively little is written about the relationship between pain and ASD in the pain literature [33]. Due to communication and assessment difficulties, there is a greater likelihood that their pain may go unrecognised and untreated (e.g., [34, 35]). Another potential barrier to assessing pain in children with ASD is the prevailing belief, frequently based on anecdotal observation or clinical impression, that pain insensitivity is a common feature in children with ASD (e.g., [2, 36-40]). Parents, caregivers, and mental health professionals have reported that some children with ASD appear to withstand painful stimuli (bumps, cuts, etc.) show absence of nociceptive reflexes (e.g., absence of hand withdrawal reflex when burning oneself), or lack of protective body position in cases of broken legs or arms [41]. However, nearly all of the support for this notion of pain insensitivity is derived from anecdotal reports and limited clinical observations [24-29, 42-44]. Despite the lack of systematic studies of pain sensitivity and reactivity in ASD, the presence of pain insensitivity in ASD has been given further validation because of its inclusion as an associated feature in standard diagnostic texts. In DSM-IV and DSM-IV-TR " $\mathrm{a}$ high threshold for pain" is described $[2,45]$ while in DSMIII the "ignoring of pain" is described (APA, 1987). Not only are children with ASD considered to have "reduced pain sensitivity," but they have also been described as "not feeling pain as intensely as others" [27], having an "indifference to pain" [44] and having a "high threshold for pain" [2]. The belief that children with ASD are insensitive to pain may bias observers' judgements of pain in these children [46].

It is important to understand the behaviours observers can use to assess pain in children and adults with ASD and to understand the potential bias of pain sensitivity information on observers' judgements of pain. Over the last decade there has been a plethora of studies investigating pain expression and perception in individuals with intellectual disabilities or individuals with developmental disabilities (often the exact diagnostic nature of these groups is not specified) (i.e., [4750]). By contrast, peer-reviewed literature investigating the relationship between pain expression and perception of pain in individuals with ASD is sparse. Research on pain in children with developmental disabilities has almost exclusively relied on observational or behavioural assessment measures $[51,52]$. The present systematic review was carried out using PRISMA guidelines [53] to primarily identify and examine the evidence for the widely held belief that individuals with ASD are insensitive to pain or have a high pain threshold. Additionally, this review will examine whether individuals with ASD react or express pain differently.

\section{Method}

Internet-based bibliographic databases (PsycINFO and PubMed) were searched to access studies which examined pain in individuals with ASD only (authors reported no comorbidity in their sample). Studies which investigated pain in individuals with ASD who also had another disorder, such as mental retardation, were not included because the present review was specifically interested in the impact pure ASD has on pain sensitivity and expression of pain. Studies which investigated pain in individuals with ASD on a psychological/behavioural level were included. Numerous studies were excluded as they explored more medical issues related to ASD such as gastrointestinal symptoms (i.e., bowel inflammation and alterations in intestinal microflora).

It is commonly reported in the literature that sensory disturbances can feel painful to individuals with ASD. For instance, rain might sound like gunfire and the individual finds this so painful that they have to cover their ears. However, studies which discussed these types of painful experiences were not included as the present review is interested in what is considered to be well-known painful events to the majority of individuals, such as needles in the skin and high and cold temperature. The process of eliminating nonrelevant papers can be seen in the flowchart (following PRISMA guidelines, [53]) (Please see Figure 1 for the Flowchart). Duplicates were excluded prior to the retrieval of references. Searches on the two databases were originally conducted on October, 18, 2012 and updated on February, 6, 2013. The following search criteria were entered into PubMed: ["pain" (title/abstract) AND "autis*" (title/abstract)] which returned a total of 126 references. The following search criteria were entered into PsycINFO: ["pain" (all text) AND "autis*"] which returned a total of 148 references. Combining the abstracts returned on these two databases, there was a total of 274 abstracts. In addition to these database searches, numerous permutations of ASD and pain were entered into Google Scholar and thoroughly searched for any additional articles not found in the database searches. For instance, [Asperger AND pain]; [autism AND pain]; [ASD and pain]. These searches only returned one additional article which was an abstract of a pilot study (the findings of which have not 
Identification

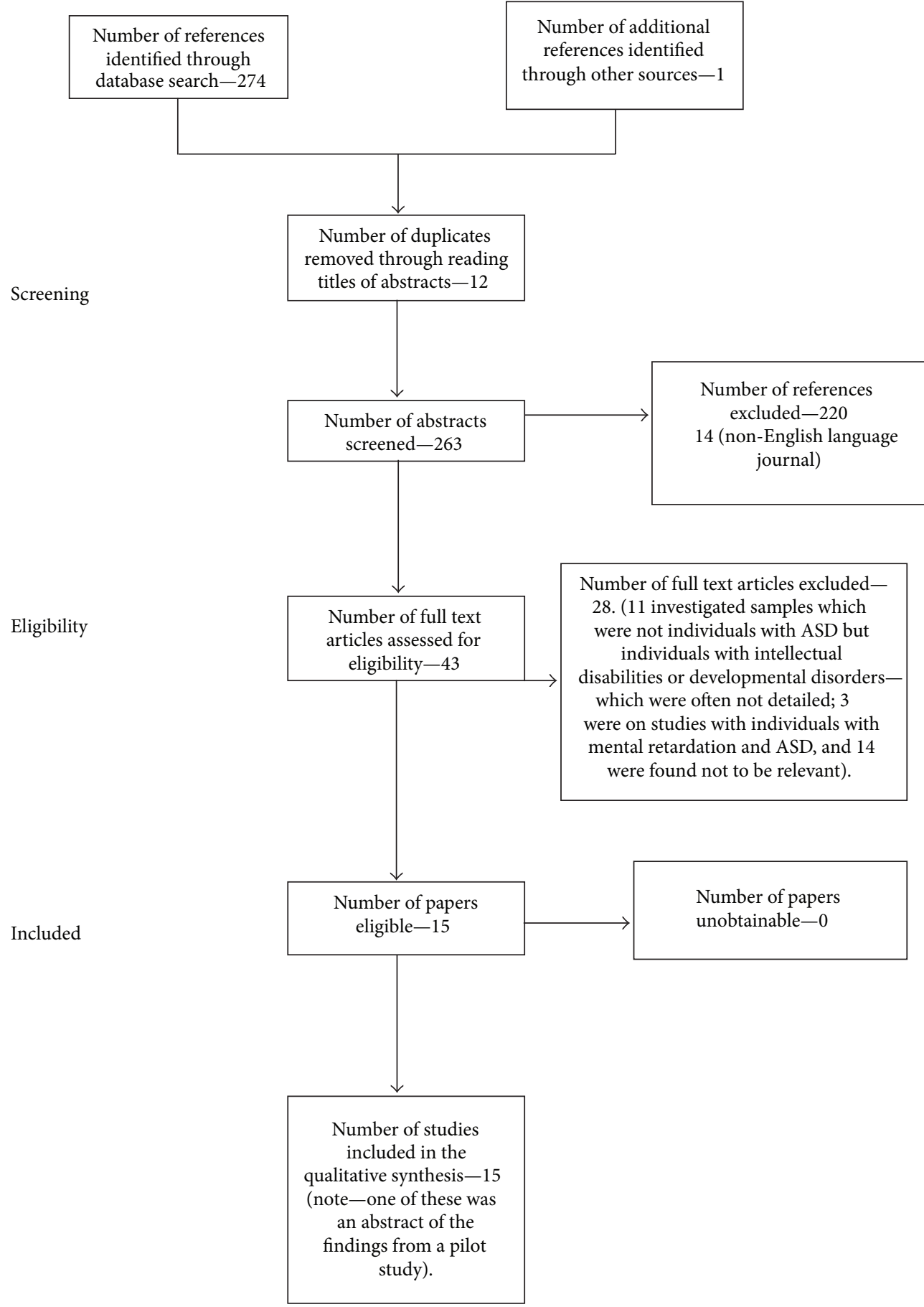

FIGURE 1: Flow of information through Systematic Review.

since been published in a peer-reviewed article to the authors knowledge).

Abstracts for each reference were obtained and screened using the following criteria.

\section{Inclusion Criteria}

(1) Human study population.
(2) Investigated psychological/behavioural pain response in individuals with ASD.

\section{Exclusion Criteria}

(1) Paper not published in English.

(2) Dissertations.

(3) Book reviews. 
(4) Studies which investigated a sample that comprised of individuals with a disorder other than ASD (for instance, developmental disabilities or intellectual disabilities) or ASD with a comorbidity (i.e., ASD and mental retardation) were excluded.

Screening. In the first stage, papers were rejected which

(i) investigated medical issues (such as intestinal inflammation) or clinical psychopharmacology aspects of ASD,

(ii) were not published in the English language.

For the next stage, papers were rejected which were not studies that involved a sample of individuals with ASD.

In addition, review papers and book chapters which were clearly reviews were excluded. Full documents were obtained for the remaining records.

\section{Results}

Five case studies and ten experimental studies were found in the PRISMA search that investigated some aspect of pain in individuals with ASD.

3.1. Case Studies. Table 1 details the five case report studies which explored pain perception, expression, or observer perception of pain in individuals with ASD.

Pain experts might be underrecognising signs and symptoms of ASD in their patients, a notion which led Bursch et al. [33] to explore this in two patients (Tony and Gregg) who displayed signs and symptoms indicative of possible ASD. Tony's mother reported a possible sensory disturbance in early childhood in that he liked to belt his trousers extremely tightly, which most children would have found painful, but Tony liked the sensation. His mother reported that he once grabbed a hot frying pan and did not seem to respond in a way typical of someone in pain. Despite both adolescents showing obvious signs and symptoms indicative of an ASD, review of previous medical records and parental interviews suggested that health care professionals did not identify the need for evaluation of these unusual characteristics. This is clinically useful to recognise because any type of pain might be exacerbated by sensory processing abnormalities and/or persistent arousal that often characterise patients with ASD $[54,55]$.

Elwin et al.[56] reviewed 17 works published in English or Swedish and 10 autobiographies to explore hyper- and hyposensitivity in individuals with ASD in the context of verbal expression. The autobiographies were written by individuals who all had an ASD diagnosis. The authors found much evidence to suggest pain insensitivity (hyposensitivity) in individuals with ASD in that pain could be indistinctly experienced. Several authors indicated that they had a very high pain threshold. For instance, one individual reported "Injuries could easily go undetected. In there, I was given a punch in the stomach, every day, though usually only one. Perhaps I was not much fun to hit because I had a very high pain threshold, and even when I did hurt I never showed what I felt. I did not know that was what you should do" [57, page 92]. They also found evidence of pain sensitivity (hypersensitivity). However, for the purposes of the present review we have excluded this as from the descriptions given in the paper by Elwin et al. [56], this was pain which was experienced as a result of sensory abonormalities not specifically related to what would typically be defined as a painful event (such as bodily injury or needle injection). They reported cases where the individual reported feeling excruciating pain racking through her head in response to a fog horn.

Rutherford [58] described the development of an infant (N.F.) who was later diagnosed with ASD in direct comparison to the development of his twin, from the prenatal period to the age of four years, through the examination of personal journals and medical records kept by the mother of the twins. Several differences in development between the twins, some as early as six months of age, were found and of particular interest was the observation that N.F. frequently showed insensitivity to pain which was exhibited as early as six months of age. Differences reemerged by the age of four years, at which point N.F. would wake up frequently during the night, sometimes as many as nine times. He would cry and yell during these times and his mother thought that he appeared to be in extreme pain.

Autoextraction of teeth (self-extraction of a tooth) is an unusual form of self-injurious behaviour (SIB) and is rarely seen in children with ASD. Ross-Russell and Sloan [17] present the case of a seven-year-old boy with mild ASD who experienced unexplained dental pain and subsequently went on to extract his own lower right deciduous canine tooth. He has also demonstrated SIB in the form of head banging. He was brought to the clinic complaining of pain of about one week's duration that was increasing in intensity, was present most of the time, and was not sensitive to hot, cold, or sweet stimulus. Within 24 hours the patient was again back at the clinic as the pain had not resolved with ibuprofen and by this time his lower right deciduous canine was very slightly mobile. The tooth had normal root anatomy and no evidence of alveolar bone loss was evident but by the next day the patient had extracted his lower right deciduous canine tooth, which was witnessed by his mother. He claimed it had been "itching" him until he got it out. This case indicates that the patient was insensitive to pain to some degree.

Mieres et al. [32] describe how a nurse and a physical therapist in an interprofessional (IP) school-based clinic worked together to meet the needs of a nine-year-old child with pervasive developmental disorder, not otherwise specified, with atypical classroom behaviours and declining student performance. The child denied pain of any type, using a 0 to 10 -point visual analogue scale. The IP team noted that the student was speaking less to other students, faculty, and staff. Although such behaviour could be a sign of withdrawal, it may also be a sign of oral problems. As five weeks had passed without any change in the student's demeanor, the child was referred to a dentist who discovered a severe abscess affecting two teeth. The dentist reported that an abscess of this size without pain is unusual, given the size, the depth, and the 
TABLE 1: Case report studies which explored pain perception, expression, or observer perception of pain in individuals with ASD.

\begin{tabular}{|c|c|c|c|}
\hline Author & Samples & $\begin{array}{c}\text { Level of functioning } \\
\text { of ASD sample }\end{array}$ & Aim of the study \\
\hline Bursch et al. [33] & $\begin{array}{l}2 \text { patients with the } \\
\text { signs and symptoms } \\
\text { of ASD. }\end{array}$ & Does not specify. & $\begin{array}{l}\text { Case report of } 2 \text { patients } \\
\text { and their sensory } \\
\text { abnormalities and pain } \\
\text { perception as observed by } \\
\text { family. }\end{array}$ \\
\hline Elwin et al. [56] & $\begin{array}{l}17 \text { works published } \\
\text { in English or } \\
\text { Swedish and } 10 \\
\text { autobiographies. }\end{array}$ & $\begin{array}{l}\text { Does not specify for } \\
\text { individuals. }\end{array}$ & $\begin{array}{l}\text { To explore hyper- and } \\
\text { hypo-sensitivity in } \\
\text { individuals with ASD in the } \\
\text { context of verbal } \\
\text { expression. Using samples } \\
\text { of published } \\
\text { autobiographies as a data } \\
\text { source. }\end{array}$ \\
\hline Mieres et al. [32] & $\begin{array}{l}9 \text { year old with } \\
\text { pervasive } \\
\text { developmental } \\
\text { disorder, not } \\
\text { otherwise specified. }\end{array}$ & Does not specify. & $\begin{array}{l}\text { To describe how a nurse } \\
\text { and a physical therapist in } \\
\text { an interprofessional (IP) } \\
\text { school-based clinic } \\
\text { collaborated to meet the } \\
\text { needs of a child with } \\
\text { PDD-NOS, with atypical } \\
\text { classroom behaviours and } \\
\text { declining student } \\
\text { performance. }\end{array}$ \\
\hline
\end{tabular}

Findings

Reported evidence of lack of pain sensitivity in both patients. That is, “...once grabbed a hot frying pan and did not seem to respond in a way typical of someone in pain."

Yes, self-reported pain insensitivity.

Pain could be indistinctly experienced, and several authors pointed out having a very high pain threshold. Injuries could easily go undetected.

Yes: parental report of pain insensitivity. For this student, pain was an unreliable indicator of both a dental infection and piercing of skin by thorny objects. Child was referred to a dentist who discovered a severe abscess affecting two teeth. The dentist reported that an abscess of this size without pain is unusual, given the size, the depth, and the proximity to bone.

Yes, suggestion that this patient had pain insensitivity.

"He has also demonstrated SIB in the form of head banging. He was brought to the clinic complaining of pain of about

Report of a case of a young child with mild ASD who presented with unexplained dental pain and who subsequently went on to extract his own lower right deciduous canine tooth. one week's duration that was increasing in intensity which was present most of the time and was not sensitive to hot, cold or sweet stimulus. Within 24 hours the patient was again back at the clinic, as the pain had not resolved with ibuprofen, and by this time his lower right deciduous canine was very slightly mobile. No evidence of alveolar bone loss and the tooth had normal root anatomy. By the next day the patient had extracted his lower right deciduous canine tooth, witnessed by his mother. He claimed it had been 'itching' him until he got it out."

Describes the development of an infant who was later diagnosed with ASD.

Twins: boy who was diagnosed with ASD

Rutherford [58] at 3 years and 1 month, and a girl who developed typically.
Directly compares his development to that of his

Does not specify. twin from a prenatal period through to the age of 4 years. Explored through examination of personal journals and medical records kept by the mother.
Yes: evidence of pain

insensitivity-maternal observations.

N.F. frequently showed insensitivity to pain which was exhibited as early as 6 to 12 months of age. 
proximity to bone. The abscess required two 10-day rounds of antibiotics until the infection was completely remedied. Two months later, the same student arrived in the clinic and stated that he was "not able to concentrate and the noise was bothering him." Again, the student denied pain, using a 0- to 10 -point pain scale, both verbal and pictorial. When given the same scale of 0 to 10 and instead asked, "How uncomfortable are you?" the student stated about a 7. Multiple superficial cuts and lacerations, some covered with bandaids, were seen bilaterally. When the mother was called from the clinic, she stated that over the weekend the student played in the sand dunes without shoes. She further stated that the other children playing with him left quickly after complaints of painful pinching in their legs and feet. The student continued to play in the dunes without any sign of discomfort and could not understand why the other children were complaining. Later, the mother discovered bleeding cuts on his feet and he was taken to a 24-hour clinic, where the physician removed 14 sand spurs. The student helped in the removal of the sand spurs without complaints of pain even when extractions were deep. However, the following morning, he complained of excessive noise, did not wish to be touched, and covered his head. The student stated at first that he had a 0 in a pain scale of 0 to 10 . However, when asked, "How uncomfortable are you?" the student indicated a 7. For this student, pain was an unreliable indicator of both a dental infection and piercing of skin by thorny objects, preventing timely treatment.

In sum, the five reported case studies all seem to provide some support to the widely held belief that individuals with ASD are insensitive to pain or have a high pain threshold.

3.2. Experimental Studies. Table 2 details the experimental studies which explored pain perception, expression, or observer perception of pain in individuals with ASD. There were ten experimental studies identified in the search of which one was an abstract presenting the findings from a pilot study. The ten studies are split up into five different sections under the subheadings of "Facial Activity to Pain Stimuli in ASD" (no. 3); "Pain Sensitivity Experienced in ASD" (no. 4); "Embodied Pain in ASD" (no. 1); "Relationship between Opioid Hormone and ASD" (no. 1) and "Oversensitivity to Pain and Age of Diagnosis of ASD” (no. 1).

3.3. Facial Activity to Pain Stimuli in ASD. Facial activity has been found to be a major determinant of observers' judgements of pain in infants [59], children [60], and adults with cognitive impairments [61]. The widely held belief that children with ASD are less sensitive to pain compared to neurotypical children may bias observers' interpretation of pain expression/behavioural reactivity in these children. Messmer et al. [46] investigated whether the perceptions of pain in children with ASD could potentially be influenced by the belief that children with ASD are insensitive to pain. Twentyseven undergraduate psychology students who had no previous experience with children with ASD were recruited at the University of British Columbia. The sample consisted of 23 females and four males, with a mean age of 20.11 years. Nineteen of the participants identified themselves as Caucasian, seven participants identified themselves as Asian, and one identified him/herself as "other." Observers received information that pain experience in children with ASD is either the same as, more intense than, or less intense than children without ASD. After viewing six video clips (which were obtained from a previous study by Nader et al. [62], described below) of children with ASD undergoing venepuncture, observers estimated pain intensity using a visual analogue scale. Venepuncture is a medical procedure which requires the use of a needle to puncture a patient's vein. Puncturing a vein provides direct access that allows for extraction of venous blood or insertion of medication or fluids directly into the blood stream and is the same basic procedure which is used to extract blood for blood donations. The sample of children with ASD used for the current study consisted of four boys and two girls between the age of three and seven. The clips consisted of the 10 seconds immediately preceding the injection and the 10 seconds immediately after needle insertion. Participants were randomly assigned to one of the three groups. Group A consisted of seven participants, and groups $\mathrm{B}$ and $\mathrm{C}$ consisted of 10 participants. Each group read a two-page booklet with information taken from "Children with Autism: A Parent's Guide Describing Features of Autism" [63]. Within the general account was a description of the pain experience of children with ASD. This description of pain in individuals with ASD was different for each group, saying either (a) "Children with autism appear to respond to pain in the same way that children without autism do"; (b) "Children with autism also appear to respond to pain differently than children without autism. In particular, they feel pain more than other children. This has been termed "pain hypersensitivity" and has recently been documented in research on children with autism"; or (c) "Children with autism also appear to respond to pain differently than children without autism. In particular, they seem to have a high tolerance for pain and do not appear to feel pain as much as other children".

After reading the booklet, participants watched six video clips of children with ASD undergoing venepuncture. The video clips had been previously coded for facial activity using the Child Facial Coding System (Child Facial Action Coding System Revised Manual, CFCS [64]). The CFCS is a facial coding system which was created as a way to assess pain experiences in children. Thirteen explicitly defined facial actions (e.g., brow lower, eye squeeze, and nose wrinkle) are coded, in terms of frequency and intensity, by a trained CFCS coder using stop-frame and slow-motion video editing equipment. After each video clip, participants rated the pain intensity of the child on a visual analogue scale (VAS), a $100 \mathrm{~mm}$ horizontal line anchored on the left by "no pain" and on the right by "worst possible pain." Participants placed a mark on the line to indicate how much pain they thought the child was feeling. The VAS is a valid measure for assessing pain intensity [65]. Mean pain intensity scores on the VAS were compared to the average facial pain activity scores from the CFCS. A Spearman rank order correlation suggested that the order of VAS ratings was highly correlated with the order of the CFCS scores ( $r s=0.943, P<0.01$ ). In sum, the main findings of this study by Messmer et al. [46] were that children who received lower scores on the CFCS were judged 


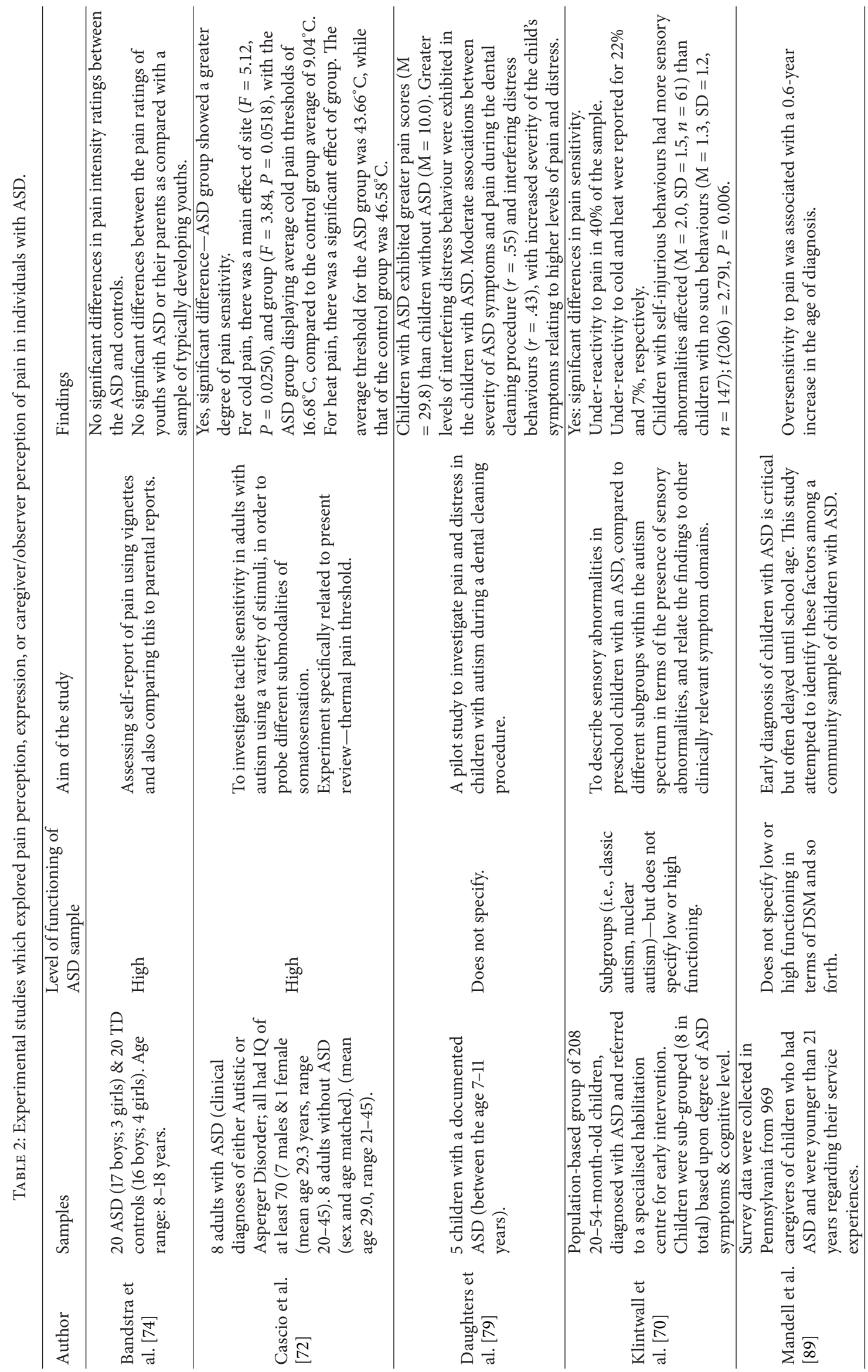




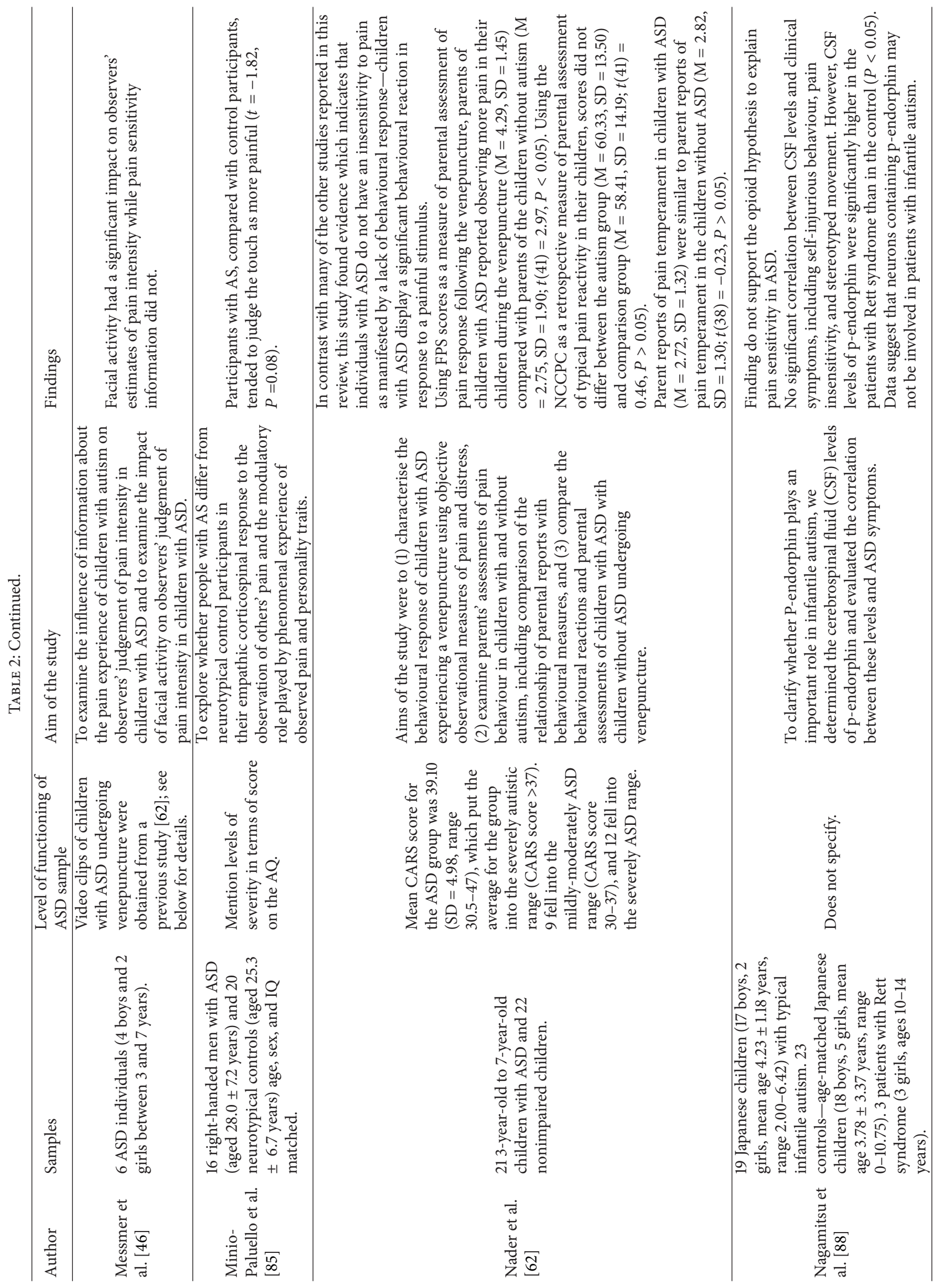




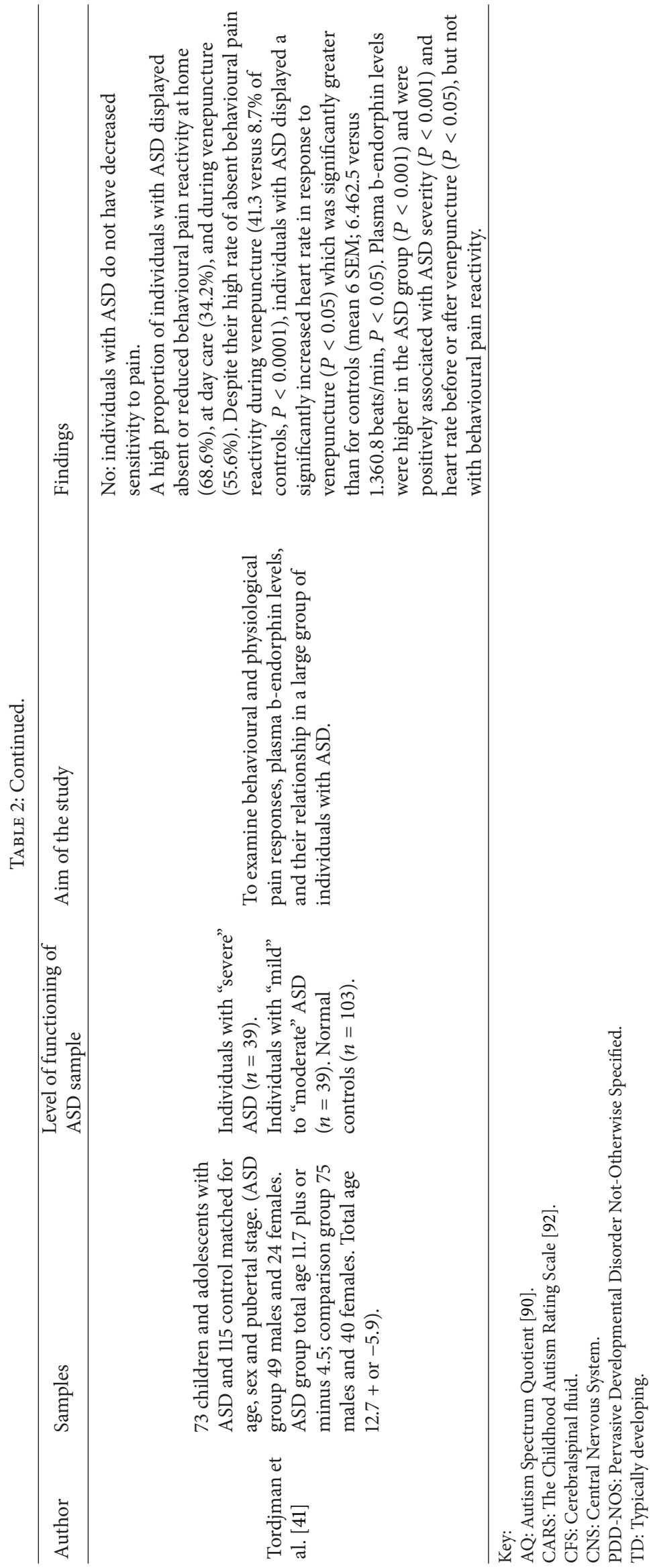


to be experiencing a lower intensity of pain and children who received higher scores on the CFCS were judged to be experiencing a higher intensity of pain. Thus, Messmer et al. [46] found that observers' ratings of pain in children with ASD were not influenced by information regarding the pain experience in children with ASD and that they were able to use facial activity as one basis for estimating pain in children with ASD. This study also indicates that the children's experience of pain is communicated, at least to some degree, through their facial activity.

Nader et al. [62] conducted a study in order to examine the behavioural response of children with ASD during venepuncture using an objective observational measure of pain and distress. In addition to this objective measure, they also examined parents' assessments of pain behaviour in children with and without ASD, including comparison of the relationship of parental reports with behavioural measures. All of these measures were compared to the same assessment conducted on control children during the same procedure. Nader et al. [62] recorded behavioural distress and facial reactions of pain in 21 three- to seven-year-old children with ASD and 22 nonimpaired children during venepuncture. Parents provided observer reports of pain and facial activity was used as an objective behavioural measure of pain. Detailed coding of videotapes were performed using the Child Facial Coding System [64] (which was the objective measure also used in the study above by Messmer et al. [46]). An objective measure of distress was also used in the present study, namely, the Observational Scale of Behavioral Distress (OSBD). The OSBD [66] is a coding system designed to assess behavioural distress in children undergoing painful medical procedures. OSBD consists of eight operationally defined behaviours indicative of anxiety and/or pain behaviour in children.

Observer reports of pain from the parents were measured using the following two procedures. Histories of pain sensitivity were assessed by asking the parents to report on prior pain reactions of their children using the Non-Communicating Children's Pain Checklist (NCCPC) [67]. Parents were also asked to provide a summary report of their child's pain temperament by responding to the following statement: "My child is very sensitive to pain of bumps or cuts or other common hurts." The parent responded to this question on a scale of $1=$ not typical/characteristic to $5=$ very typical/characteristic. Lastly, the Faces Pain Scale (FPS; [68]) was given to the parents. This consists of seven faces showing gradual increases in pain expression from left to right (neutral to pain). The parents were asked to select the face that they felt represents the degree of pain experienced by their child during the venepuncture procedure.

Findings from the study by Nadar et al. [62] revealed that the behavioural responses of the children with ASD were overall similar to the comparison group, except the substantial facial pain reactivity instigated by the venepuncture in the children with ASD exceeded that found in the control group. The degree of concordance between parental report and observed pain responses were consistently better for the comparison group. For the ASD group, no significant correlation was observed between the FPS scores provided by the parents and the facial pain responses of the children, $r=-0.154, P>0.05$. Interestingly, children with ASD who had been assessed by their parents as having a lower pain sensitivity and reactivity tended to show greater facial reactions and behavioural distress in response to the venepuncture. Using FPS scores as a measure of parental assessment of pain response following the venepuncture, parents of children with ASD reported observing more pain in their children during the venepuncture $(M=4.29, \mathrm{SD}=1.45)$ compared with parents of the children without ASD $(M=2.75, \mathrm{SD}=$ $1.90 ; t(41)=2.97, P<0.05)$. Using the NCCPC as a retrospective measure of parental assessment of typical pain reactivity in their children, scores did not differ between the ASD group $(M=60.33, \mathrm{SD}=13.50)$ and comparison group $(M=58.41, \mathrm{SD}=14.19 ; t(41)=0.46, P>0.05)$. Parent reports of pain temperament in children with ASD $(M=$ $2.72, \mathrm{SD}=1.32$ ) were similar to parent reports of pain temperament in the children without $\operatorname{ASD}(M=2.82, \mathrm{SD}=1.30$; $t(38)=-0.23, P>0.05)$. In addition, although the ASD severity of the ASD group was well characterised and ranged from mild to severe, there was no information about level of intellectual functioning for this group. Overall, these findings demonstrate that children with ASD can display a significant behavioural reaction in response to a painful stimulus which is in contrast to the widely held belief in the literature that individuals with ASD are insensitive to pain. However, the study also shows that some of the caregivers did not interpret their child's pain expression accurately. Children with ASD, who had been assessed by their parents as having a lower pain sensitivity and reactivity, tended to show greater facial reactions and behavioural distress in response to the venepuncture. However, this is difficult to draw strong conclusions from this since it may be that the event was simply more distressing for the individuals with ASD rather than that they had any greater degree of pain sensitivity.

In another study, Tordjman et al. [41] examined behavioural and physiological pain responses, plasma beta-endorphin levels and their relationship in 73 children and adolescents with autism and 115 normal individuals matched for age, sex, and pubertal stage during blood drawing. Pain reactivity was assessed for patients in three different observational situations. (1) in day care, where two caregivers independently rated overall pain reactivity on a daily basis during the month preceding the blood drawing; (2) at home, where parents rated pain-related behaviour during the same month as the caregivers. In this situation, there were enough daily life situations involving pain to distinguish reactions to a variety of types of noxious and painful stimuli such as being burned, having internal pain (tooth pain, ear infection, headache, etc.), and other accidental painful stimuli (cutting, pinching, banging, etc.); (3) during the blood drawing at a medical centre, when a direct clinical observation was conducted by a nurse and child psychiatrist not belonging to the caregiver team. Normal controls were similarly assessed for pain reactivity to the venepuncture using the Pre-Linguistic Behavioral Pain Reactivity Scale (PL-BPRS) [69]. The scale looks at five different pain scenarios, namely, (1) paradoxical pain reactivity, the apparent pleasure reaction to a painful stimulus (such as smiling or laughing); (2) absence of pain reactivity, 
absence of nociceptive reflexes (such as absence of hand withdrawal reflex when burning oneself or absence of arm withdrawal reflex from the needle during a blood drawing); (3) hyporeactivity to pain, incomplete pain reactivity or abnormally delayed reaction time to painful stimulus; (4) normal pain reactivity such as cries, screams, moaning, grimaces, reflexes of nociceptive withdrawal, lack of movement, body orientation, and glance towards the painful area, and lastly, (5) hyperreactivity to pain, disproportionate cries, and screams given the painful stimulus (with hypersensitive light touch). A checklist was used to indicate the presence or absence of SIB, aggressive behaviours directed against others, stereotyped behaviours, and social withdrawal during the blood drawing situation. Physiological measures included plasma b-endorphin levels analysis and a heart rate measurement to examine cardiovascular response to the blood drawing (with a stethoscope placed on the thorax considering that some patients can react negatively when their wrist is touched) immediately before and after the venepuncture (15second measurement period).

Tordjman et al. [41] found that across the three observational situations, abnormal behavioural responses to painful stimuli were highly prevalent in individuals with ASD of low to moderate functioning. In general, there was a shift to hyporeactive or absent pain reactions in the ASD group. A high proportion of individuals with ASD displayed absent or reduced behavioural pain reactivity at home (68.6\%), at day care $(34.2 \%)$ and during venepuncture (55.6\%). Although this pattern of observed behaviour is consistent with a number of previous studies, most prior reports did not distinguish pain reactivity from pain sensitivity. It is critical to keep this distinction in mind and not to conclude that absence of behavioural pain reactivity means absence of pain sensitivity. Despite their high rate of absent behavioural pain reactivity during venepuncture $(41.3 \%$ versus $8.7 \%$ of controls, $P<0.0001$ ), individuals with ASD displayed a significantly increased heart rate in response to venepuncture $(P<0.05)$. This response (Delta heart rate) was significantly greater than for controls (mean \pm SEM; $6.4 \pm 2.5$ versus $1.3 \pm$ 0.8 beats $/ \mathrm{min}, P<0.05$ ). This strongly indicates that prior reports of reduced pain sensitivity in ASD are related to a different mode of pain expression rather than to an insensitivity or endogenous analgesia. Plasma beta-endorphin levels were higher in the ASD group $(P<0.001)$ and were positively associated with ASD severity $(P<0.001)$ and heart rate before or after venepuncture $(P<0.05)$, but not with behavioural pain reactivity. This is inconsistent with the opioid theory of ASD that would suggest that high levels of plasma beta-endorphin is associated with behavioural pain reactivity. In addition to the physiological response to the venepuncture, behavioural changes following the venepuncture or other painful stimuli occurring at home and day hospital (SIB, aggressive behaviours, stereotyped behaviours, social withdrawal) also suggest that children with ASD perceive pain, but do not express it in the same way that control children do.

The findings by Tordjman et al. [41] also show that a significant proportion of individuals with ASD did not display low/absent overall pain reactivity according to the parental, caregiver, and blood drawing evaluations. In fact, the majority (78\%) of individuals with ASD were actually found to exhibit normal behavioural reactivity to burning; highlighting the importance of distinguishing different types of painful stimuli. Lastly, 22\% of individuals with ASD displayed normal behavioural pain reactivity to the venepuncture and $15.9 \%$ displayed hyperreactivity which is in agreement with Nader et al. [62]. In sum, this study indicates that there may be different subgroups within the ASD population. One subgroup may experience pain insensitivity, another pain sensitivity, and the other normal pain sensitivity. However, there are numerous factors to consider when making such a conclusion at this early stage and this is outlined in the discussion. For instance, this present study found that the majority of individuals with ASD exhibited normal pain reactivity to burning. It may be that individuals with ASD may need to experience a particular high level of pain such as burning before they express normal pain reactivity. However, when the painful event is not so severe some individuals with ASD may have difficulty in expressing the pain.

3.4. Pain Sensitivity Experienced in ASD. Klintwall et al. [70] investigated sensory abnormalities in a populationbased group of 208 20-54-month-old children, diagnosed with ASD and referred to a specialised habilitation centre for early intervention. Children were subgrouped (eight in total) based upon degree of autistic symptoms and cognitive level by a research team at the centre. Parents were interviewed systematically about any abnormal sensory reactions in the child. In the whole group, pain and hearing were the most commonly affected modalities. An interview according to the PARIS schedule (developed by Gillberg and colleagues within the "Paris Autism Research In Sib-pairs" study, [71]) was performed with one of the parents. This interview included structured questions about the child's sensory reactions to light, sound, smell, and so forth. However, for the purposes of this review their results for underreactivity to pain, underreactivity to heat, and underreactivity to cold are reported. Only clinically significant sensory abnormalities were scored as "present" in the study. Children in the most typical ASD subgroup (nuclear autism with no learning disability) had the highest number of affected modalities. There were no group differences in number of affected sensory modalities between groups of different cognitive levels or level of expressive speech, supporting the notion that sensory abnormality is very common in young children with ASD and providing further justification for inclusion of this symptom in the diagnostic criteria for ASD in the upcoming DSM-V. From the total group of 208 children, at least one type of major sensory abnormality was registered in 158 individuals (76\%). The most commonly reported sensory abnormality was overreactivity to sound (44\%) and underreactivity to pain (40\%). Underreactivity to cold and heat was reported for $22 \%$ and $7 \%$, respectively. Interestingly, children with self-injurious behaviours had a greater number of affected sensory abnormalities $(M=2.0, \mathrm{SD}=1.5, n=61)$ compared to children with no such self-injurious behaviours $(M=1.3, \mathrm{SD}=1.2, n=$ $147) ; t(206)=2.791, P=0.006$. Therefore, this study provides some support to the widely held belief that many 
individuals (40\%) with ASD are insensitive (under reactive) to pain.

Cascio et al. [72] recruited eight adults with highfunctioning ASD (clinical diagnoses of either Autistic Disorder or Asperger Disorder; DSM-IVTR; [2]); there were seven males and one female (mean age 29.3 years, range $20-$ 45). Eight adults without ASD were recruited from the community, selected to match each individual with autism on age and gender (mean age 29.0 years, range $21-45$ ). Each participant completed a brief questionnaire, the Adult Sensory Profile [73] to determine whether groups differed in terms of their experience with sensory stimuli in everyday life. Cascio et al. [72] compared tactile sensation in adults with ASD compared to controls on two sites of the body: (1) the hairy skin of the right dorsal forearm and (2) the glabrous skin of the right thenar palm. A variety of tactile sensations were investigated. However, for the purposes of this review only those that were pain related are reported here. These were the thermal sensation-cold pain and heat pain. Participants were instructed to respond as soon as the stimulation reached a point of being "painfully or uncomfortably hot (or cold)." In order to alleviate any anxiety about the pain stimuli, participants were reminded that the device was limited to temperatures that are too mild to produce skin damage, and that their response triggered the return of the thermode to its baseline temperature. For cold pain, there was a main effect of site $(F=5.12, P=0.0250)$, and group $(F=3.84, P=0.0518)$, with the ASD group displaying average cold pain thresholds of $16.68^{\circ} \mathrm{C}$, compared to the control group average of $9.04^{\circ} \mathrm{C}$. For heat pain, there was a significant effect of group $(F=6.79$, $P<0.01)$, and site $(F=7.37, P=0.0073)$, and a significant group $x$ session interaction $(F=8.18, P=0.0048)$. The average threshold for the ASD group was $43.66^{\circ} \mathrm{C}$, while that of the control group was $46.58^{\circ} \mathrm{C}$. Overall, the ASD group showed a greater degree of pain sensitivity to thermal pain at both sites recorded in this study as this group's cold and heat pain thresholds were lower compared to the control group.

Bandstra et al. [74] examined self-reported and parentreported pain in 20 high-functioning youths with ASD (17 boys; 3 girls) and 20 typically developing controls (16 boys; 4 girls) ranging in age from 8 to 18 years and matched on age and IQ. This is the first study to assess the self-report of pain, using vignettes, in high-functioning children and adolescents with ASD. The Charleston Pediatric Pain Pictures (CPPP) are a series of 17 cartoon pictures depicting scenes of medical, play, and home situations [75]. Each drawing has a central figure of a young non-sex-specific child lacking facial expression, who is engaged in an activity. Thirteen of the 17 scenarios depict pain-provoking events and each has a short verbal vignette that describes the event taking place in the picture. One example of the 13 pain scenarios was: "You touched the hot stove and burned your hand. Show me how much hurt you would have". The amount of pain the participants would expect to feel was self-reported using the Faces Pain Scale-Revised (FPS, [76] and a Numeric Rating Scale (NRS) in a series of validated hypothetical pain situations depicted in cartooned images (e.g., scraping knee on pavement). The FPS- $\mathrm{R}$ is comprised of 5 line drawings of faces, presented horizontally, representing increasing levels of pain, typically from no pain (0) to extreme pain (10). In addition to the FPS-R, participants and their parents were asked to rate the pain of the hypothetical situations using an NRS. The NRS was provided using a 0 to 5 scale not only to ensure simplicity of the task for children in the study, but also to provide participants with the same number of response options as provided in the FPS-R. So children and adolescents were asked to provide ratings of their hypothetical pain using both the FPS-R and the NRS, whereas parents were asked to only provide ratings of their child's pain using the NRS. Findings revealed no differences between the pain ratings of youths with ASD or their parents as compared with a sample of typically developing youths.

The lack of differences in pain intensity ratings between the ASD and control youths in the study by Bandstra et al. [74] conflicts with other recent findings which showed greater facial and behavioural pain responses during painful medical procedures [41, 62]. Discrepancies between different measures of pain (e.g., behavioural versus self-report measures) are not unusual [77]; therefore it is possible that the selfreport data obtained in this study represent a unique perspective on the subjective pain experience for youths with ASD. It is also possible that youths with ASD, although experiencing comparable levels of pain as typically developing children (as evidenced by the current data), express their pain in a more behaviourally and facially reactive manner (as evidenced in prior research). However, despite these issues, Bandstra et al. [74] highlighted the potential confounders that may have been present in the other major studies which did find increased facial pain response to the individuals with ASD. Specifically the study by Nader et al. [62] was confounded by its use of a bundling procedure (wrapping the child in a blanket for the purpose of constricting movement during the procedure) in preparation for the venupuncture procedure for the group of individuals with ASD and not the control group, a difference which could have accounted for the significantly greater pain responses evidenced by the children with ASD as compared with the controls in that study [62]. Also a recent study showing greater behavioural response in children with ASD also found higher levels of a physiological marker for stress in the ASD sample [41]. This indicates the possibility that the increased behavioural reactivity may not be an expression of pain; rather they are distressed at undergoing a medical procedure. Another important factor to consider in trying to understand pain in individuals with ASD is the degree of functioning of this group, which varies across the studies. For instance, Tordjman et al. [41] used a sample of nonverbal and low-functioning individuals with ASD, while Nader et al. [62] omitted any information about the level of functioning in their sample. The study by Bandstra et al. [74], on the other hand, used a high-functioning sample of children and adolescents with ASD. Also, Bandstra et al. [74] investigated pain responses in children aged 8 to 18 years, which represents a significantly older age group than the participants included in the study by Nader et al. [62]), for example, and aspects of the pain experience (e.g., ability to provide self-report) are known to change as typically developing children grow older [78]. Similar to the youth ratings, no differences emerged between the ASD and control groups for 
parent ratings of the amount of pain they would expect their children to show. However, this finding does not necessarily mean that youths with ASD express their pain in the same way as typically developing youths. Rather, parents of the children in this group may have grown accustomed to their children's idiosyncratic pain expressions (e.g., angry responses) over time and have learned to interpret their child's cues accurately. Although group averages for parent and child ratings were similar, additional correlations demonstrated a lack of concordance between parent and child dyads which is not a surprising phenomenon in paediatric pain assessment. This finding may even provide further support for the argument that the pattern between parent and child pain ratings is consistent regardless of whether or not the child has ASD. Furthermore, the lack of concordance in ASD is important as it indicates that, as with typically developing children, it is important for clinicians to gather pain intensity ratings from youths with ASD, rather than only relying on parent report.

In their abstract, Daughters et al. [79] report their findings of a pilot study they carried out to examine pain and distress experienced by children with ASD during a dental cleaning procedure. The authors hypothesised that children with ASD would exhibit greater levels of behavioural distress and pain during the dental procedure compared to control children. Five children with a diagnosis of ASD and four control children participated in the study (ages 7-11 years) scheduled for a dental cleaning procedure (without sedation) took part in the pilot study. Prior to the dental cleaning procedure, caregivers were asked to complete a behavioural checklist to identify the severity of the child's stereotyped behaviours, social interaction, and communication difficulties. The dental cleaning procedures were videotaped and were later coded using a variation of the Brief Behavioral Distress Scale (BBDS), an observational measure of children's procedurerelated distress. After the dental cleaning procedure, the caregivers were asked to complete the Non-Communicating Children's Pain Checklist-Revised (NCCPC) in order to assess their child's pain. The findings revealed that the mean pain scores during the dental procedure were indicative of pain for both groups. However, the children with ASD exhibited greater pain scores $(M=29.8)$ than children without ASD $(M=10.0)$. Greater levels of interfering distress behaviour were exhibited in the children with ASD compared to children without ASD. There were also moderate associations between severity of ASD symptoms and pain during the dental cleaning procedure $(r=0.55)$ and interfering distress behaviours $(r=0.43)$, with increased severity of the child's symptoms relating to higher levels of pain and distress. In sum, this pilot study indicates that individuals with ASD are more sensitive to pain during dental cleaning procedures.

3.5. Embodied Pain in ASD. Observing emotions or bodily sensations in another individual produces brain activations largely overlapping those which are activated during the direct experience of the same feelings. This overlap in activated brain regions between observed and directly experienced emotions or bodily sensations indicates that empathic brain responses may rely on resonant, mirror-like systems [80-82]. The idea that empathy for pain may be mediated by mirror systems emerged with the finding that neurons in the anterior cingulate cortex (ACC) fire in response to both pain in the self and the observation of pain in another [83]. Although ASD are often described in terms of reduced empathic abilities [84], evidence for reduced empathy in domains different from mentalising and perspective taking (for instance pain) is sparse. To investigate this, MinioPaluello et al. [85] used a sample of sixteen right-handed men with Asperger's Syndrome (a type of ASD) (aged $28.0 \pm 7.2$ years) and 20 neurotypical controls (aged $25.3 \pm 6.7$ years) age, sex, and IQ matched.

Minio-Paluello et al. [85] used single-pulse transcranial magnetic stimulation (TMS) to explore a rudimentary form of empathy, called "sensorimotor contagion", elicited in neurotypical participants when they observe painful stimuli applied to the body of another person. The authors regard sensorimotor contagion to have taken place when there is a reduction of corticospinal excitability recorded from the specific body part that is vicariously affected by the observed painful stimulation, in this case, the hand muscles. This inhibition to observation of pain inflicted on another body is characteristic of the corticospinal inhibition found during actual noxious stimulation (when the pain is directly inflicted). So in the study carried out by Minio-Paluello et al. [85], participants underwent single-pulse TMS during observation of painful and nonpainful stimuli affecting another individual. Motor-evoked potentials (MEPs) induced by focal single-pulse TMS of the left primary motor cortex (M1) were simultaneously recorded from two right-hand muscles, the first dorsal interosseous (FDI), and the abductor digiti minimi (ADM). Four types of video clips (each lasting $1.8 \mathrm{sec}-$ onds) were presented on a 19-inch screen. Video clips were (1) "static": static right hand; (2) "Pain": needle deeply penetrating the FDI muscle; (3) "Touch": cotton swab gently touching the FDI region; and (4) "Tomato": needle deeply penetrating a tomato. Thus, whereas participants' FDI muscle was vicariously involved by the painful stimulation, the ADM muscle served as a somatotopic control because it was not shown to be penetrated. Previous studies of TMS show that watching moving body parts or hands increased corticospinal excitability. In order to eliminate this confounding effect, the hands in the video were static in the clips and the syringe holder was not visible. In addition to these objective neurophysiological measures, participants were also asked to imagine how the pain would feel, if applied to them. The qualities of the imagined pain were measured using the McGill Pain Questionnaire (MPQ) [86], which is made up of Sensory (items 1-10, 17-19) and Affective (items 11-15, 20) subscales, and through the Hurts value, a rating between 0 and 10 indicating how much the participants thought the injection would hurt them.

Minio-Paluello et al. [85] found that when observing other's pain, participants with ASD, in contrast to neurotypical control participants, did not show any amplitude reduction of motor-evoked potentials recorded from the muscle vicariously affected by pain, nor did their neurophysiological response correlate with imagined pain sensory qualities. All experimental video clips were similarly rated by the two groups ( $p s>0.10$ ) except for the Static condition, which was significantly less arousing for ASD $(P<0.02)$. Participants 
with ASD, compared with control participants, perceived themselves less able to identify with the model being touched ( $t=2.07, P=0.050)$ and tended to judge the touch as more painful $(t=-1.82, P=0.08)$. When asked to imagine how they would feel if receiving the painful stimulation shown in the videos and to rate the sensory and affective qualities of imagined pain, control participants and individuals with ASD gave similar ratings (all $p s>0.33$ ). Therefore the lack of sensorimotor contagion in ASD cannot be explained by group differences in the imagined "painfulness" of the observed events. They were therefore able to correctly understand or identify how painful a particular event would be despite showing abnormal neurophysiological responses. Although participants with ASD did not embody others' pain, the observation of painful stimuli inflicted to the hand muscle of another person inhibited control participants' corticospinal representation of the same muscle (i.e., the FDI muscle). Those MEPs recorded from the ADM muscle are not modulated cannot be explained in the terms of reduced reactivity of this muscle. Indeed, when videos depict the ADM being penetrated by a needle, similar corticospinal inhibition of this muscle has been observed [87]. In sum, finding no embodiment of others' pain or reduced empathic abilities in individuals with ASD (as evidenced by reduced or absent sensorimotor contagion during the observation of pain affecting another person, the hand in the movie clips) provides neurophysiological evidence for reduced empathic resonance in people with ASD and suggests that their difficulties with empathy is mediated not only by cognitive dimensions but also by sensorimotor resonance with others.

3.6. Relationship between Opioid Hormone and ASD. Nagamitsu et al. [88] measured cerebralspinal fluid (CSF) levels of beta-endorphin, an opioid hormone, in 19 Japanese children (17 boys, 2 girls, mean age 4.23 years, range $2.00-$ 6.42) with typical infantile autism (ASD). Some children presented with accessory symptoms such as self-injurious behaviour (3/19), pain insensitivity (8/19), and stereotyped movements (10/19). The controls consisted of 23 age-matched Japanese children (18 boys, 5 girls, mean age 3.78 years, range 0-10.75) who had undergone lumbar puncture for the diagnosis of a possible central nervous system (CNS) infection but whose CSF showed normal results. CSF levels of p-endorphin in three patients with the Rett syndrome (3 girls, ages 10-14 years) who presented with symptoms resembling those of infantile ASD were also recorded. In infantile autism, CSF levels of beta-endorphin did not differ significantly from those of age-matched controls. No significant correlation between CSF levels and clinical symptoms, including selfinjurious behaviour, pain insensitivity, and stereotyped movement was found. However, CSF beta-endorphin levels were significantly higher in the patients with Rett syndrome than in the control $(P<0.05)$. Findings indicated that neurons containing beta-endorphin may not be involved in patients with infantile autism, therefore not supporting the relationship between dysfunction of brain opioid and ASD.

3.7. Oversensitivity to Pain and Age of Diagnosis of ASD. Mandell et al. [89] attempted to identify factors which may delay diagnosis of ASD among a community sample of children with ASD. Survey data was collected in Pennsylvania from 969 caregivers of children who had ASD and were younger than 21 years regarding their service experiences. The average age of diagnosis was 3.1 years for children with autistic disorder, 3.9 years for pervasive developmental disorder not otherwise specified, and 7.2 years for Asperger's disorder (a type of ASD). Interestingly, oversensitivity to pain was associated with a 0.6 -year increase in the age of diagnosis. The association of oversensitivity to pain with later diagnosis may be because this symptom prompts clinicians to search for other organic causes and not consider developmental issues.

3.8. Level of Functioning in ASD Group across Case and Experimental Studies. Of the five case studies only one specifies level of functioning and was mild ASD [17]. The remaining four case studies do not indicate level of functioning [32, 33, $56,58]$. Of the ten experimental studies, two studies include high functioning individuals with ASD $[72,74]$. Three studies did not specify level of functioning $[70,79,88]$. One did not specify low or high functioning using clinical guidelines and examined a variety of ASD symptoms to determine a level of functioning but does not report how many are contained within each category [89]. Another study, rather than defining in terms of high and low functioning, describes levels of severity based on scores on the Autism Spectrum Quotient (AQ, [90]) [85]. Two studies used the same data and employed The Childhood Autism Rating Scale (CARS, [91]) to create two groups: severely autistic and mildly-moderately autistic and the majority of the group fell into the severely autistic range $[46,62]$. Lastly, another study had 39 patients in the severe ASD group and 39 in the "mild" to "moderate" ASD group, assessing ASD severity using the Autism Diagnostic Interview-Revised (ADI-R, [92]). [41]. Four of the experimental studies did not include a comparison/control group $[46,70,79,89]$.

\section{Discussion}

Five case studies and ten experimental studies were found in the PRISMA search which investigated some aspect of pain in individuals with ASD. All five case studies described individuals with ASD who were exhibiting pain insensitivity [17, 32, 33, 56, 58]. The two cases presented by Bursch et al. [33] demonstrate how chronic pain can be the focal symptom and perseverative focus of attention for individuals with an ASD. Once focused on pain, difficulties in shifting attentional focus can serve to increase pain and associated distress. Therefore, implementing a treatment that somehow interrupts the perseveration might reduce or even eliminate the pain that the individual experiences. In fact, this is exactly what Zeltzer and Schlank [93] found. They describe a case in their pain clinic where a child with ASD presented to them yelling repeatedly, "Ow! Ow! Ow!" Two months earlier, the child had sustained a leg injury and he had been shouting ever since. Zeltzer and Schlank suggested that rather than shouting, the child should replace this by squeezing a ball instead. Interestingly, this resulted in a transfer of his expression to the point where he actually reported feeling better because he no longer felt 
embarrassed about repeatedly shouting "Ow!". These findings strongly indicate that treating the perseveration can be the most effective way to reduce suffering for some patients [7]. What has also been proposed by numerous researchers is that in children with ASD the anxiety often experienced by this population might actually contribute to their pain experience. This holds especially true for those individuals whose muscles remain tense for extensive durations [93].

Also important for clinical practice is the case study by Mieres et al. [32] which suggests a particular approach is required in assessing the subjective feeling of pain in individuals with ASD. Rather than ask how much pain they are feeling, they suggest instead a new series of questions, the key being "How uncomfortable are you?" (used in addition to the verbal and pictorial pain scale).

Of the ten experimental studies only one found no significant difference in pain sensitivity between patients with ASD and controls. Specifically, no significant differences were found between the pain ratings of youth with ASD or their parents as compared with a sample of typically developing youths [74]. Interestingly, overall, only one of the experimental studies (compared to all five of the case studies) found evidence of underreactivity to pain (suggestive of pain insensitivity) in $40 \%$ of their sample [70]. One study [41] found that individuals with ASD do not have a decreased sensitivity for pain and investigated both behavioural reactivity to pain during venepuncture as well as plasma b-endorphin concentrations and heart rate. The additional physiological measures were particularly important since there was an absence of any behavioural pain reactivity in the individuals with ASD during venepuncture despite their higher heart rate and plasma b-endorphin levels-strongly suggesting that the individuals with ASD were not insensitive to pain.

Five studies found evidence of a greater degree of pain sensitivity in individuals with ASD $[62,72,79,85,89]$. Interestingly, oversensitivity to pain was associated with a 0.6 -year increase in the age of diagnosis [89]. One study [46] investigated the influence of information about the pain experience of children with ASD on observers' judgement of pain intensity in children with ASD and examined the impact of facial activity on observers' judgement of pain intensity in children with ASD. Facial activity was found to have a significant impact on observers' estimates of pain intensity; pain sensitivity information did not [46]. This is in contrast to the view that parents' ratings of pain in their children with ASD may be distorted due to misinformation about pain insensitivity in their children [62]. A possible limitation with this study, in terms of investigating the effect of information of pain sensitivity in individuals with ASD, is that the students in this study had no personal relationship to the individuals they observed and rated in the videos. This might have contributed to the lack of effect of pain information on the judgments made. A different picture might emerge if the same situation was applied to individuals with a personal relationship to the child such as a parent. These results have important implications for the assessment and management of pain in children with ASD [46]. The finding that observers may be able to decode pain information from facial activity [46] is important because children with ASD frequently lack the skills to express their pain verbally and this could put them at risk for substandard health care. The findings of a significant behavioural reaction in response to a painful stimulus in individuals with ASD [62, 72, 79, 85, 89] contradict the widespread belief in the literature of pain insensitivity in individuals with ASD. Some of the findings reported in this review also question the clinical appropriateness of parental global report as an assessment tool for pain in children with ASD [62]. Another study investigated whether P-endorphin plays an important role in infantile autism by measuring the cerebrospinal fluid (CSF) levels of p-endorphin and evaluated the correlation between these levels and ASD symptoms [88]. Findings did not support the opioid hypothesis to explain pain sensitivity in ASD [88].

Another important aspect is whether the five experimental studies that included different levels of functioning or severity of ASD $[41,46,62,85,89]$ report whether this had any notable effect on the results. For instance, were the findings weaker for individuals who are higher functioning. Two studies did find differences $[41,85]$ while three did not $[46,62$, 89]. Tordjman et al. [41] found that plasma b-endorphin levels were positively associated with ASD severity. Minio-Paluello et al. [85] found that corticospinal inhibition was maximal in the individuals with fewer ASD traits.

\subsection{Expression of Pain in Individuals with ASD. Numerous} anecdotal reports show that caregivers frequently describe unusual, or absent, responses to painful stimuli in their children with ASD. Some caregivers are even able to describe unique behaviours in their child that enable them to know when they are in pain. However, it is crucial to point out here that altered pain expression is not universally observed in ASD. Despite this most experts are in agreement that the pain experience appears different in individuals with ASD [94].

Tordjman et al. [41] argue that their findings indicate that prior reports of reduced pain sensitivity in ASD are related, not to an insensitivity or endogenous analgesia to pain but to a different mode of pain expression. This is without doubt the most crucial finding and clearly further investigation to explore this aspect is required. The findings by Tordjman et al. [41] constitute a clear challenge to theories of reduced pain sensitivity in ASD since they found that painful stimuli can produce physical and psychic stress in individuals with ASD and that this stress can be manifested by physiological responses and expressed through autistic behaviours. Tordjman et al. [41] hypothesise that the different mode of pain expression in individuals with ASD may be mediated by (1) verbal communication impairments, (2) deficits in nonverbal communication and body image problems (difficulty locating the painful area), or (3) other cognitive problems such as (a) difficulty in establishing cause-effect relationships between the pain sensation and the stimulus causing the pain, (b) problems discriminating, representing and identifying sensations and emotions which involves abstraction and symbolisation capacities (the perception of pain integrates sensorial, emotional, and cognitive factors [95]), (c) problems of learning socially appropriate responses to pain [41].

The majority of the experimental studies included in the review examine pain reaction to a specific medical 
procedure venepuncture. Therefore, the findings cannot be generalised to other contexts and pain situations such as pain during everyday situations and experience of chronic pain in individuals with ASD. It is also important to acknowledge that the experiences of children with ASD occur along a spectrum of severity. Therefore it is highly possible that the experience and expression of pain may differ depending on where the individual lies on this spectrum (Messmer et al. [46]); the level of communicative and language abilities of individuals [94] and the impact of different ASD diagnoses (i.e., Asperger's disorder; PDD-NOS; Rett syndrome) on pain expression and reactivity. This variability again emphasises that interventions and treatments must be tailored to each specific child [94].

Despite the complexity in interpreting the findings from the sparse amount of studies to date which have looked at pain sensitivity in individuals with ASD, what is clear is that there is a need for a pain assessment tool specifically for use in this population [94]. Existing instruments may be inappropriate. To my knowledge, there has only been one study (from a dissertation submitted to the University of Florida for the degree of Doctor of Philosophy) which has attempted to identify whether there are unique pain indicators applicable to a significant amount of children with ASD and whether there are pain indicators identified by caregivers that are completely unique to the one child. Inglese [94] found several objective, observable and measurable indicators which were presumed by the author to be relevant to pain assessment in ASD (i.e., "furrowed brow," "banging his/her head," "injuring oneself," "grimacing," "guarding," and "increased heart rate"), many other identified indicators were subjective and required (a) knowledge of the child's baseline, and (b) monitoring for changes from normal (i.e., "crankiness," "being less active," "rocking unusually," "acting "off”, and "irritability"). Inglese [94] suggests then that in the designing of a new instrument specifically for pain assessment in individuals with ASD, there needs to be the inclusion of sections which are objective and quantifiable - these could be used by individual(s) who are unfamiliar to the child being assessed-in addition to sections which are based on caregiver judgments regarding what is typical in their child. In effect encompassing both caregiver information regarding their child's baseline and objective assessment questions which can be conducted by individuals unfamiliar with the child is crucially important in creating a comprehensive pain assessment in this population [94].

4.2. Limitations. Sensations are often thought to be logically private, subjective, self-intimating, and the source of incorrigible knowledge for those who have them. Since pain is often thought to be a "subjective" experience, this has lead researchers to use the report as the gold standard for pain experience. Many of the studies identified in the present review investigate pain reactivity using caregiver reports which have obvious limitations such as reporting bias. However, the most notable limitation with this is highlighted by research which suggests that parents of children with ASD perform worse than parents of control children in facial emotion task. For instance, in an emotional labelling task in response to schematic facial patterns representing five basic emotions, parents of children with ASD performed worse than parents of control children (i.e., [96]). The study by Nader et al. [62] (one of the studies reported in this review) is consistent with the difficulties found in parents of children with ASD in correctly interpreting facial emotions. Nader et al. [62] found that some of the caregivers did not interpret their child's pain expression accurately in that children with ASD who had been assessed by their parents as having a lower pain sensitivity and reactivity tended to show greater facial reactions and behavioural distress in response to the venepuncture.

Similarly, there is the issue of asking individuals with ASD to rate pain according to facial expressions of pain (i.e., Faces Pain Scale (FPS)). Numerous researchers maintain that individuals with ASD have difficulty with processing facial expression (i.e., [97]). Individuals with ASD process faces differently and show reduced attention to faces and facial expressions [98]. This reduced interest in faces is likely to impair their face processing skills, so that children with ASD do not become "face experts" like their typically developing peers [99].

The majority of the small number of studies which investigated some aspects of pain specifically in individuals with ASD are limited by their sample size. Seven of the ten experimental studies had sample sizes of ASD groups less than 21 . The remaining three had 73, 969, and 208 individuals with ASD. More studies with larger sample sizes examining behavioural reactivity to pain as well as measuring physiological responses are imperative to our understanding of pain experience in individuals with ASD. A further complication in attempting to draw conclusions from the literature to date are the differences across studies in terms of cognitive development of the individuals with ASD (not to mention the wide differences in age). For instance, in one study [41] all patients were cognitively impaired: mean full scale IQ $=42.2$, $\mathrm{SD}=3.2$ (range 40-58) while another study all had IQ of at least 70 [72]. These limitations and differences pose a significant problem when trying to determine whether there is pain insensitivity, greater pain sensitivity or no difference in individuals with ASD.

Lastly, it is also difficult to disentangle from the experimental studies conducted to date as to how much of the observed pain reactions, and so forth were due to the individuals with ASD level of distress rather than any pain response. Individuals with ASD might display more distress during the venepuncture procedure compared to controls and this might be completely independent from how painful they find the experience.

4.3. Future Directions and Clinical Implications. Further studies are needed to recognise illnesses earlier in the absence of pain or pain perception in children with an ASD and to develop reliable and valid metrics for pain identification for both verbal and nonverbal individuals with ASD [32]. Further research is also needed to explore the concordance between parent report of pain sensitivity and observed reactivity of children with ASD to every day painful incidents. Children with ASD may exhibit typical behavioural reactions to procedural pain in a clinical setting but atypical responses 
to everyday pain in their home environment [62]. It is important to understand how different types of pain in different settings are perceived in order to acceptably manage pain in children with ASD. Additionally, more research is needed to understand how observers decode the pain experience of children with ASD and explore the potentially biasing effect of pain sensitivity information on observers' estimates of pain in children with ASD [46]. The study by Mieres et al. [32] also stresses the importance of the way in which clinicians ask patients with ASD about how much pain they are experiencing. They found that asking the individual with ASD how uncomfortable they were on a scale of 1 to 10 was a more accurate representation of the pain they felt than when they were asked directly how much pain they were in on a scale of 1 to 10 .

Further research is also need to explore further how children and adults with ASD react to various types (acute versus chronic) or degrees of pain. The need for this research is emphasised by the study reported in this review [41] that a significant number of individuals with ASD reported absent or reduced pain reactivity ( $41.3 \%$ versus $8.7 \%$ of controls) but in the same sample the majority (78\%) exhibited normal pain reactivity to burning based on caregiver report. This clearly shows that the type and severity of the pain event is important when studying pain in this population.

In a study just published, Wager et al. [100], based on four studies involving a total of 114 participants, developed an fMRI-based measure that predicts pain intensity at the level of the individual person and found that it is possible to use fMRI to assess pain elicited by noxious heat in healthy persons. Given the limitations of the experimental studies to date (for instance the reliability of self-report measures of pain and caregiver report of pain in their child), this would be an more robust and objective way to investigate whether pain experience is any different in individuals with ASD compared to controls. This might also have the potential to show whether there is a discordance between neural signatures of pain and the expression of pain in the individual with ASD.

\section{Conclusions}

There is still relatively little research on the unique problems posed by the expression of pain and sensitivity to painful stimuli in individuals with intellectual and developmental disabilities [101] and particularly in individuals with ASD from childhood to adulthood. Overall, the findings reported here of a significant behavioural reaction in response to a painful stimulus in individuals with ASD contradict the wide spread belief in the literature of pain insensitivity in individuals with ASD. The case studies all reported pain insensitivity in individuals with ASD and provide an example of how impaired sensory perceptions can mask and delay the ability of health care professionals to recognise the need for treatment. However, the majority of the ten experimental studies reviewed here indicate that the idea that individuals with ASD are pain insensitive needs to be challenged. This systematic review highlights the need for a shift away from the widely and long-held belief that children and adults with ASD have a reduced pain sensitivity, do not feel pain as intensely as others, and have an indifference to pain and a high threshold for pain. What was also highlighted by some of the findings of this review is the importance of further study to explore the theory that the pain expression in individuals with ASD differs from that of neurotypicals. Recognition of all these findings have important implications for the treatment and recognition of the need for treatment in individuals with ASD.

\section{Conflict of Interests}

The author declares no conflict of interests.

\section{References}

[1] C. P. Johnson and S. M. Myers, "Identification and evaluation of children with autism spectrum disorders," Pediatrics, vol. 120, no. 5, pp. 1183-1215, 2007.

[2] American Psychiatric Association, Diagnostic and Statistical Manual of Mental Disorders, American Psychiatric Association, Washington, DC, USA, 4th edition, 2000.

[3] G. T. Baranek, F. J. David, M. D. Poe, W. L. Stone, and L. R. Watson, "Sensory experiences questionnaire: discriminating sensory features in young children with autism, developmental delays, and typical development," Journal of Child Psychology and Psychiatry and Allied Disciplines, vol. 47, no. 6, pp. 591-601, 2006.

[4] Centers for Disease Control and Prevention, "Prevalence of autism spectrum disorders-autism and developmental disabilities monitoring network, 14 Sites, United States, 2008," Morbidity and Mortality Weekly Report, vol. 61, no. SS03, pp. 1-19, 2012.

[5] E. Courchesne and K. Pierce, "Why the frontal cortex in autism might be talking only to itself: local over-connectivity but longdistance disconnection," Current Opinion in Neurobiology, vol. 15, no. 2, pp. 225-230, 2005.

[6] T. Grandin, Thinking in Pictures, Doubleday, New York, NY, USA, 1995.

[7] D. J. Simons and P. W. Land, "Early experience of tactile stimulation influences organization of somatic sensory cortex," $\mathrm{Na}$ ture, vol. 326, no. 6114, pp. 694-697, 1987.

[8] W. Dunn, B. S. Myles, and S. Orr, "Sensory processing issues associated with Asperger syndrome: a preliminary investigation," American Journal of Occupational Therapy, vol. 56, no. 1, pp. 97-102, 2002.

[9] G. Iarocci and J. McDonald, "Sensory integration and the perceptual experience of persons with autism," Journal of Autism and Developmental Disorders, vol. 36, no. 1, pp. 77-90, 2006.

[10] S. J. Rogers and S. Ozonoff, "Annotation: what do we know about sensory dysfunction in autism? A critical review of the empirical evidence," Journal of Child Psychology and Psychiatry, and Allied Disciplines, vol. 46, no. 12, pp. 1255-1268, 2005.

[11] A. Baghdadli, C. Pascal, S. Grisi, and C. Aussilloux, "Risk factors for self-injurious behaviours among 222 young children with autistic disorders," Journal of Intellectual Disability Research, vol. 47 , no. 8, pp. 622-627, 2003.

[12] J. L. Matson and T. T. Rivet, "The effects of severity of autism and PDD-NOS symptoms on challenging behaviors in adults with intellectual disabilities," Journal of Developmental and Physical Disabilities, vol. 20, no. 1, pp. 41-51, 2008.

[13] J. L. Matson and T. T. Rivet, "Characteristics of challenging behaviours in adults with autistic disorder, PDD-NOS, and 
intellectual disability," Journal of Intellectual and Developmental Disability, vol. 33, no. 4, pp. 323-329, 2008.

[14] J. Rojahn, J. Wilkins, J. L. Matson, and J. Boisjoli, “A comparison of adults with intellectual disabilities with and without ASD on parallel measures of challenging behaviour: the behavior problems inventory-01 (BPI-01) and autism spectrum disordersbehavior problems for intellectually disabled adults (ASDBPA)," Journal of Applied Research in Intellectual Disabilities, vol. 23, no. 2, pp. 179-185, 2010.

[15] K. R. M. Smith and J. L. Matson, "Behavior problems: differences among intellectually disabled adults with co-morbid autism spectrum disorders and epilepsy," Research in Developmental Disabilities, vol. 31, no. 5, pp. 1062-1069, 2010.

[16] F. Furniss and A. B. Biswas, "Recent research on aetiology, development and phenomenology of self-injurious behaviour in people with intellectual disabilities: a systematic review and implications for treatment," Journal of Intellectual Disability Research, vol. 56, no. 5, pp. 453-475, 2012.

[17] M. Ross-Russell and P. Sloan, "Autoextraction in a child with autistic spectrum disorder," British Dental Journal, vol. 198, no. 8, pp. 473-474, 2005.

[18] F. J. Barrera, J. M. Teodoro, T. Selmeci, and A. Madappuli, "Selfinjur, pain, and the endorphin theory," Journal of Developmental and Physical Disabilities, vol. 6, no. 2, pp. 169-192, 1994.

[19] C. A. Sandman, "B-endorphin disregulation in autistic and selfinjurious behavior: a neurodevelopmental hypothesis," Synapse, vol. 2, no. 3, pp. 193-199, 1988.

[20] C. A. Sandman, M. A. Spence, and M. Smith, "Proopiomelanocortin (POMC) disregulation and response to opiate blockers," Mental Retardation and Developmental Disabilities Research Reviews, vol. 5, no. 4, pp. 314-321, 1999.

[21] F. J. Symons, "Pain and self-injury: mechanisms and models," in Self-Injurious Behavior: Genes, Brain, and Behavior, S. Schroeder, T. Thompson, and M. L. Oster-Granite, Eds., American Psychological Association, 2002.

[22] W. E. MacLean, R. C. Tervo, J. Hoch, M. Tervo, and F. J. Symons, "Self-Injury among a community cohort of young children at risk for intellectual and developmental disabilities," Journal of Pediatrics, vol. 157, no. 6, pp. 979-983, 2010.

[23] Nutrition Health Review, "Autism linked to increased pain," Nutrition Health Review: The Consumer's Medical Journal, no. 95, p. 5, 2006.

[24] J. Panksepp, "A neurochemical theory of autism," Trends in Neurosciences, vol. 2, no. 7, pp. 174-177, 1979.

[25] J. Panksepp and T. L. Sahley, "Possible brain opioid involvement in disrupted social intent and language development of autism," in Neurobiological Issues in Autism, E. Schopler and G. B. Mesibov, Eds., pp. 357-372, Plenum Press, New York, NY, USA, 1987.

[26] E. Frescka and K. L. Davis, "The opioid model in psychiatric research," in Neuropeptides and Psychiatric Disorders, C. B. Nemeroff, Ed., pp. 169-191, American Psychiatric Press, Washington, DC, USA, 1991.

[27] C. Gillberg, "The role of endogenous opioids in autism and possible relationships to clinical features," in Aspects of Autism: Biological Research, L. Wing, Ed., pp. 31-37, Gaskell/The National Autistic Society, London, UK, 1988.

[28] L. Sher, "Autistic disorder and the endogenous opioid system," Medical Hypotheses, vol. 48, no. 5, pp. 413-414, 1997.

[29] J. W. Kalat, "Speculations on similarities between autism and opiate addiction," Journal of Autism and Childhood Schizophrenia, vol. 8, no. 4, pp. 477-479, 1978.
[30] C. Gillberg, "Endogenous opioids and opiate antagonists in autism: brief review of empirical findings and implications for clinicians," Developmental Medicine and Child Neurology, vol. 37, no. 3, pp. 239-245, 1995.

[31] M. Seid, M. Sherman, and A. B. Seid, "Perioperative psychosocial interventions for autistic children undergoing ENT surgery," International Journal of Pediatric Otorhinolaryngology, vol. 40, no. 2-3, pp. 107-113, 1997.

[32] A. C. Mieres, V. Smallwood, and S. K. Nicholson, "Retrospective case report: evaluation of pain in a child with pervasive developmental disorder," Pediatric Physical Therapy, vol. 23, no. 2, pp. 194-200, 2011.

[33] B. Bursch, K. Ingman, L. Vitti, P. Hyman, and L. K. Zeltzer, "Chronic pain in individuals with previously undiagnosed autistic spectrum disorders," Journal of Pain, vol. 5, no. 5, pp. 290 295, 2004.

[34] K. L. Hadden, C. von Baeyer, and K. D. Craig, "Pain in children with severe cognitive and communication impairment: commentary," Pediatric Pain Letter, vol. 4, pp. 4-6, 2000.

[35] P. Stallard, L. Williams, S. Lenton, and R. Velleman, "Pain in cognitively impaired, non-communicating children," Archives of Disease in Childhood, vol. 85, no. 6, pp. 460-462, 2001.

[36] C. Gillberg, L. Terenius, and G. Lonnerholm, "Endorphin activity in childhood psychosis. Spinal fluid levels in 24 cases," Archives of General Psychiatry, vol. 42, no. 8, pp. 780-783, 1985.

[37] J. E. Mauk, "Autism and pervasive developmental disorders," Pediatric Clinics of North America, vol. 40, no. 3, pp. 567-578, 1993.

[38] G. T. Baranek and G. Berkson, "Tactile defensiveness in children with developmental disabilities: responsiveness and habituation," Journal of Autism and Developmental Disorders, vol. 24, no. 4, pp. 457-471, 1994.

[39] M. O’Neill and R. S. P. Jones, "Sensory-perceptual abnormalities in autism: a case for more research?" Journal of Autism and Developmental Disorders, vol. 27, no. 3, pp. 283-294, 1997.

[40] T. Peeters and C. Gillberg, Autism: Medical and Educational Aspects, Whurr, London, UK, 2nd edition, 1999.

[41] S. Tordjman, G. M. Anderson, M. Botbol et al., "Pain reactivity and plasma $\beta$-endorphin in children and adolescents with autistic disorder," PLoS One, vol. 4, no. 8, Article ID e5289, 2009.

[42] H. I. Kaplan, B. J. Sadock, and J. A. Grebb, Kaplan and Sadock's Synopsis of Psychiatry: Behavioral Sciences, Clinical Psychiatry, Williams and Wilkins, Baltimore, Md, USA, 7th edition, 1994.

[43] M. Prior and J. S. Werry, "Autism, schizophrenia, and allied disorders," in Psychopathological Disorders of Childhood, H. C. Quay and J. S. Werry, Eds., pp. 156-210, John Wiley, New York, 1986.

[44] L. Wing, The Autistic Spectrum, Constable, London, UK, 1996.

[45] American Psychiatric Association, Diagnostic and Statistical Manual of Mental Disorders, American Psychiatric Association, Washington, DC, USA, 4th edition, 1994.

[46] R. L. Messmer, R. Nader, and K. D. Craig, "Brief report: judging pain intensity in children with autism undergoing venepuncture: the influence of facial activity," Journal of Autism and Developmental Disorders, vol. 38, no. 7, pp. 1391-1394, 2008.

[47] K. K. Biersdorff, "Incidence of significantly altered pain experience among individuals with developmental disabilities," American Journal on Mental Retardation, vol. 98, no. 5, pp. 619-631, 1994.

[48] K. A. Mercer, Expression of emotion by infants with and without disabilities [Ph.D. thesis], University of Central Lancashire, UK, 2000. 
[49] L. M. Breau and C. S. Camfield, "Pain disrupts sleep in children and youth with intellectual and developmental disabilities," Research in Developmental Disabilities, vol. 32, no. 6, pp. 28292840, 2011.

[50] B. Temple, C. Dubé, D. McMillan et al., "Pain in people with developmental disabilities: a scoping review," Journal on Developmental Disabilities, vol. 18, no. 1, pp. 73-86, 2012.

[51] L. M. Breau, C. S. Camfield, P. J. McGrath, and G. A. Finley, "The incidence of pain in children with severe cognitive impairments," Archives of Pediatrics and Adolescent Medicine, vol. 157, no. 12, pp. 1219-1226, 2003.

[52] M. van Dijk, A. Valkenburg, A. A. Boerlage, D. Tibboel, and J. S. Veerkamp, "Children with intellectual disabilities and pain perception: a review and suggestions for future assessment protocols," European Archives of Paediatric Dentistry, vol. 10, no. 2, pp. 57-60, 2009.

[53] A. Liberati, D. G. Altman, J. Tetzlaff et al., "The PRISMA statement for reporting systematic reviews and meta-analyses of studies that evaluate healthcare interventions: explanation and elaboration," British Medical Journal, vol. 339, p. b2700, 2009.

[54] E. Courchesne, A. J. Lincoln, R. Yeung-Courchesne, R. Elmasian, and C. Grillon, "Pathophysiologic findings in nonretarded autism and receptive developmental language disorder," Journal of Autism and Developmental Disorders, vol. 19, no. 1, pp. $1-17,1989$.

[55] S. J. Hutt, C. Hutt, D. Lee, and C. Ounsted, "A behavioural and electroencephalographic study of autistic children," Journal of Psychiatric Research, vol. 3, no. 3, pp. 181-197, 1965.

[56] M. Elwin, L. Ek, A. Schröder, and L. Kjellin, "Autobiographical accounts of sensing in Asperger syndrome and high-functioning autism," Archives in Psychiatric Nursing, vol. 26, no. 5, pp. 420-429, 2012.

[57] G. Gerland, A Real Person: Life on the Outside, Souvenir Press, London, UK, 1997.

[58] M. D. Rutherford, "A retrospective journal-based case study of an infant with autism and his twin," Neurocase, vol. 11, no. 2, pp. 129-137, 2005.

[59] H. D. Hadjistavropoulos, K. D. Craig, R. V. E. Grunau, and C. C. Johnston, "Judging pain in newborns: facial and cry determinants," Journal of Pediatric Psychology, vol. 19, no. 4, pp. 485491, 1994.

[60] L. M. Breau, P. J. McGrath, K. D. Craig, D. Santor, K. L. Cassidy, and G. J. Reid, "Facial expression of children receiving immunizations: a principal components analysis of the child facial coding system," Clinical Journal of Pain, vol. 17, no. 2, pp. 178-186, 2001.

[61] D. L. LaChapelle, T. Hadjistavropoulos, and D. C. Kenneth, "Pain measurement in persons with intellectual disabilities," Clinical Journal of Pain, vol. 15, no. 1, pp. 13-23, 1999.

[62] R. Nader, T. F. Oberlander, C. T. Chambers, and K. D. Craig, "Expression of pain in children with autism," Clinical Journal of Pain, vol. 20, no. 2, pp. 88-97, 2004.

[63] M. D. Powers, Children with Autism: A Parent's Guide, Woodbine House, Rockville, Md, USA, 1989.

[64] C. T. Chambers, K. L. Cassidy, P. J. McGrath, C. A. Gilbert, and K. D. Craig, Child Facial Coding System Revised Manual, Dalhousie University, Halifax, Canada, University of British Columbia, Vancouver, Canada, 1996.

[65] M. P. Jensen and P. Karoly, "Self-report scales and procedures for assessing pain in adults," in Handbook of Pain Assessment, D. C. Turk and R. Melzack, Eds., pp. 15-34, Guildford, New York, NY, USA, 2nd edition, 2001.
[66] S. M. Jay and C. Elliott, Observation Scale of Behavioral Distress-Revised Manual, Children's Hospital of Los Angeles, Los Angeles, Calif, USA, 1986.

[67] P. J. McGrath, C. Rosmus, C. Canfield, M. A. Campbell, and A. Hennigar, "Behaviours caregivers use to determine pain in nonverbal, cognitively impaired individuals," Developmental Medicine and Child Neurology, vol. 40, no. 5, pp. 340-343, 1998.

[68] D. Bieri, R. A. Reeve, G. D. Champion, L. Addicoat, and J. B. Ziegler, "The faces pain scale for the self-assessment of the severity of pain experienced by children: development, initial validation, and preliminary investigation for ratio scale properties," Pain, vol. 41, no. 2, pp. 139-150, 1990.

[69] S. Tordjman, C. Antoine, D. J. Cohen et al., "Study of the relationships between self-injurious behavior and pain reactivity in infantile autism," Encéphale, vol. 25, no. 2, pp. 122-134, 1999.

[70] L. Klintwall, A. Holm, M. Eriksson et al., "Sensory abnormalities in autism: a brief report," Research in Developmental Disabilities, vol. 32, no. 2, pp. 795-800, 2011.

[71] E. Fernell, Å. Hedvall, F. Norrelgen et al., "Developmental profiles in preschool children with autism spectrum disorders referred for intervention," Research in Developmental Disabilities, vol. 31, no. 3, pp. 790-799, 2010.

[72] C. Cascio, F. McGlone, S. Folger et al., "Tactile perception in adults with autism: a multidimensional psychophysical study," Journal of Autism and Developmental Disorders, vol. 38, no. 1, pp. 127-137, 2008.

[73] W. Dunn, "The sensations of everyday life: empirical, theoretical, and pragmatic considerations," American Journal of Occupational Therapy, vol. 55, no. 6, pp. 608-620, 2001.

[74] N. F. Bandstra, S. A. Johnson, J. H. Filliter, and C. T. Chambers, "Self-reported and parent-reported pain for common painful events in high-functioning children and adolescents with autism spectrum disorder," Clinical Journal of Pain, vol. 28, no. 8, pp. 715-721, 2012.

[75] R. W. Belter, J. A. McIntosh, A. J. Finch Jr, and C. F. Saylor, "Preschoolers' ability to differentiate levels of pain: relative efficacy of three self-report measures," Journal of Clinical Child Psychology, vol. 17, no. 4, pp. 329-335, 1988.

[76] C. L. Hicks, C. L. Von Baeyer, P. A. Spafford, I. Van Korlaar, and B. Goodenough, "The faces pain scale-revised: toward a common metric in pediatric pain measurement," Pain, vol. 93, no. 2, pp. 173-183, 2001.

[77] J. E. Beyer, P. J. McGrath, and C. B. Berde, "Discordance between self-report and behavioral pain measures in children aged 3-7 years after surgery," Journal of Pain and Symptom Management, vol. 5, no. 6, pp. 350-356, 1990.

[78] B. Goodenough, L. Kampel, G. D. Champion et al., "An investigation of the placebo effect and age-related factors in the report of needle pain from venipuncture in children," Pain, vol. 72, no. 3, pp. 383-391, 1997.

[79] H. Daughters, T. Palermo, and J. Koh, "Procedural pain and distress in children with autism-a pilot study," Journal of Pain, vol. 8, no. 4, Supplement 1, p. S31, 2007.

[80] S. Leiberg and S. Anders, "The multiple facets of empathy: a survey of theory and evidence," Progress in Brain Research, vol. 156, pp. 419-440, 2006.

[81] S. D. Preston and F. B. M. de Waal, "Empathy: its ultimate and proximate bases," Behavioral and Brain Sciences, vol. 25, no. 1, pp. 1-20, 2002.

[82] J. Decety and P. L. Jackson, "The functional architecture of human empathy," Behavioral and Cognitive Neuroscience Reviews, vol. 3, no. 2, pp. 71-100, 2004. 
[83] C. S. Allely, "I feel your pain!'Neurological and anecdotal evidence to suggest we really can feel others'pain," The Psychologist, vol. 25, no. 2, pp. 160-161, 2012.

[84] S. Baron-Cohen and S. Wheelwright, "The empathy quotient: an investigation of adults with asperger syndrome or high functioning autism, and normal sex differences," Journal of Autism and Developmental Disorders, vol. 34, no. 2, pp. 163-175, 2004.

[85] I. Minio-Paluello, S. Baron-Cohen, A. Avenanti, V. Walsh, and S. M. Aglioti, "Absence of embodied empathy during pain observation in Asperger syndrome," Biological Psychiatry, vol. 65, no. 1, pp. 55-62, 2009.

[86] R. Melzack, "The McGill pain questionnaire: major properties and scoring methods," Pain, vol. 1, no. 3, pp. 277-299, 1975.

[87] A. Avenanti, I. M. Paluello, I. Bufalari, and S. M. Aglioti, "Stimulus-driven modulation of motor-evoked potentials during observation of others' pain," NeuroImage, vol. 32, no. 1, pp. 316$324,2006$.

[88] S. Nagamitsu, T. Matsuishi, T. Kisa et al., "CSF $\beta$-endorphin levels in patients with infantile autism," Journal of Autism and Developmental Disorders, vol. 27, no. 2, pp. 155-163, 1997.

[89] D. S. Mandell, M. M. Novak, and C. D. Zubritsky, "Factors associated with age of diagnosis among children with autism spectrum disorders," Pediatrics, vol. 116, no. 6, pp. 1480-1486, 2005.

[90] S. Baron-Cohen, S. Wheelwright, R. Skinner, J. Martin, and E. Clubley, "The Autism-Spectrum Quotient (AQ): evidence from Asperger syndrome/high-functioning autism, males and females, scientists and mathematicians," Journal of Autism and Developmental Disorders, vol. 31, no. 1, pp. 5-17, 2001.

[91] C. Lord, M. Rutter, and A. L. Couteur, "Autism diagnostic interview-revised: a revised version of a diagnostic interview for caregivers of individuals with possible pervasive developmental disorders," Journal of Autism and Developmental Disorders, vol. 24, no. 5, pp. 659-685, 1994.

[92] E. Schopler, R. J. Reichler, and B. R. Renner, The Childhood Autism Rating Scale, Western Psychological Services, Los Angeles, Calif, USA, 7th edition, 1998.

[93] L. K. Zeltzer and C. B. Schlank, Conquering your Child's Chronic Pain: A Pediatrician's Guide for Reclaiming a Normal Childhood, HarperCollins, New York, NY, USA, 2005.

[94] M. D. Inglese, Pain expression in children with autism spectrum disorder (ASD), A foundation for instrument development [Ph.D. thesis], The Graduate School of the University of Florida, 2008.

[95] S. W. G. Derbyshire and P. Bennett, "Fetal stress responses," Lancet, vol. 344, no. 8922, p. 615, 1994.

[96] M. T. Palermo, P. Pasqualetti, G. Barbati, F. Intelligente, and M. P. Rossini, "Recognition of schematic facial displays of emotion in parents of children with autism," Autism, vol. 10, no. 4, pp. 353-364, 2006.

[97] M. J. Smith, B. Montagne, D. I. Perrett, M. Gill, and L. Gallagher, "Detecting subtle facial emotion recognition deficits in highfunctioning Autism using dynamic stimuli of varying intensities," Neuropsychologia, vol. 48, no. 9, pp. 2777-2781, 2010.

[98] G. Dawson, K. Toth, R. Abbott et al., "Early social attention impairments in autism: social orienting, joint attention, and attention to distress," Developmental Psychology, vol. 40, no. 2, pp. 271-283, 2004.

[99] G. Dawson, S. J. Webb, and J. McPartland, "Understanding the nature of face processing impairment in autism: insights from behavioral and electrophysiological studies," Developmental Neuropsychology, vol. 27, no. 3, pp. 403-424, 2005.
[100] T. D. Wager, L. T. Atlas, M. A. Lindquist, M. Roy, W. ChoongWan, and E. Kross, "An fMRI-based neurologic signature of physical pain," The New England Journal of Medicine, vol. 368, no. 15, pp. 1388-1397, 2013.

[101] R. Melzack and S. K. Burns, "Neurophysiological effects of early sensory restriction," Experimental Neurology, vol. 13, no. 2, pp. 163-175, 1965. 


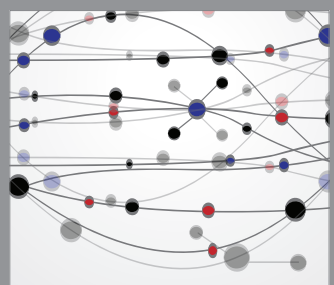

The Scientific World Journal
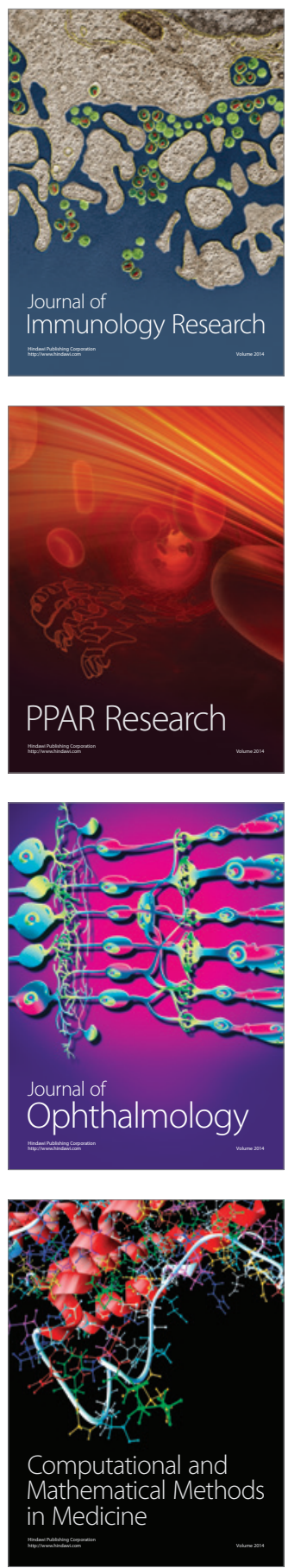

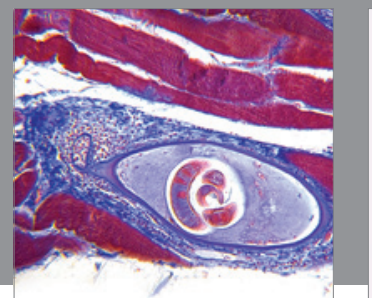

Gastroenterology

Research and Practice
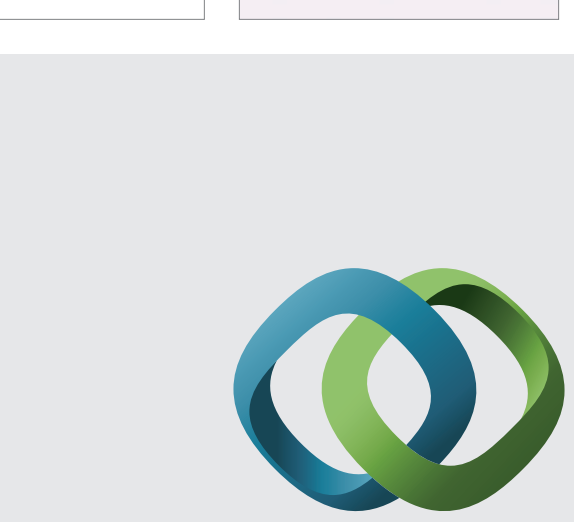

\section{Hindawi}

Submit your manuscripts at

http://www.hindawi.com
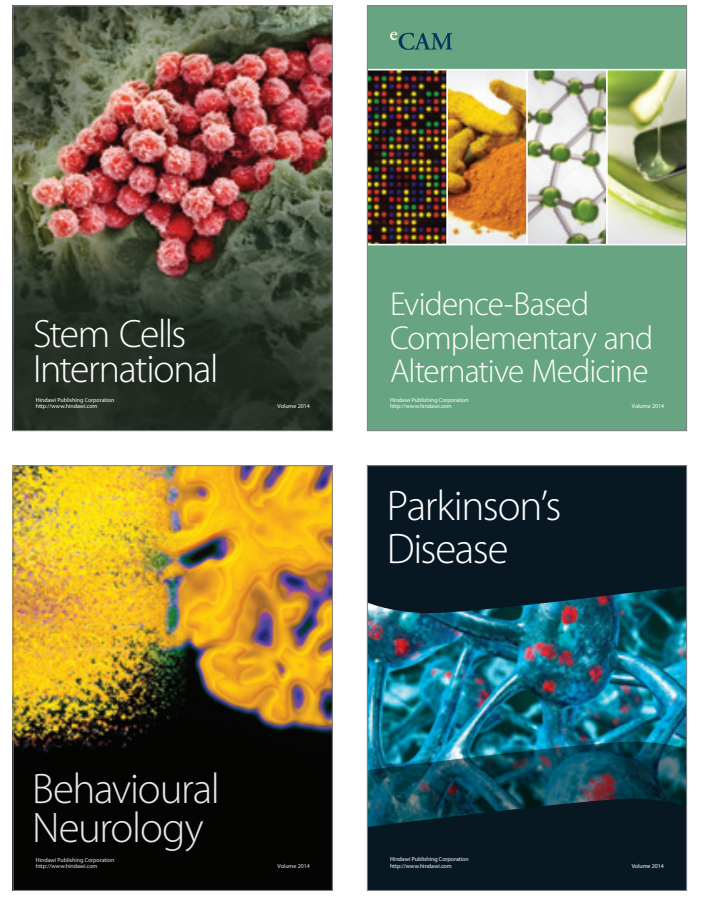
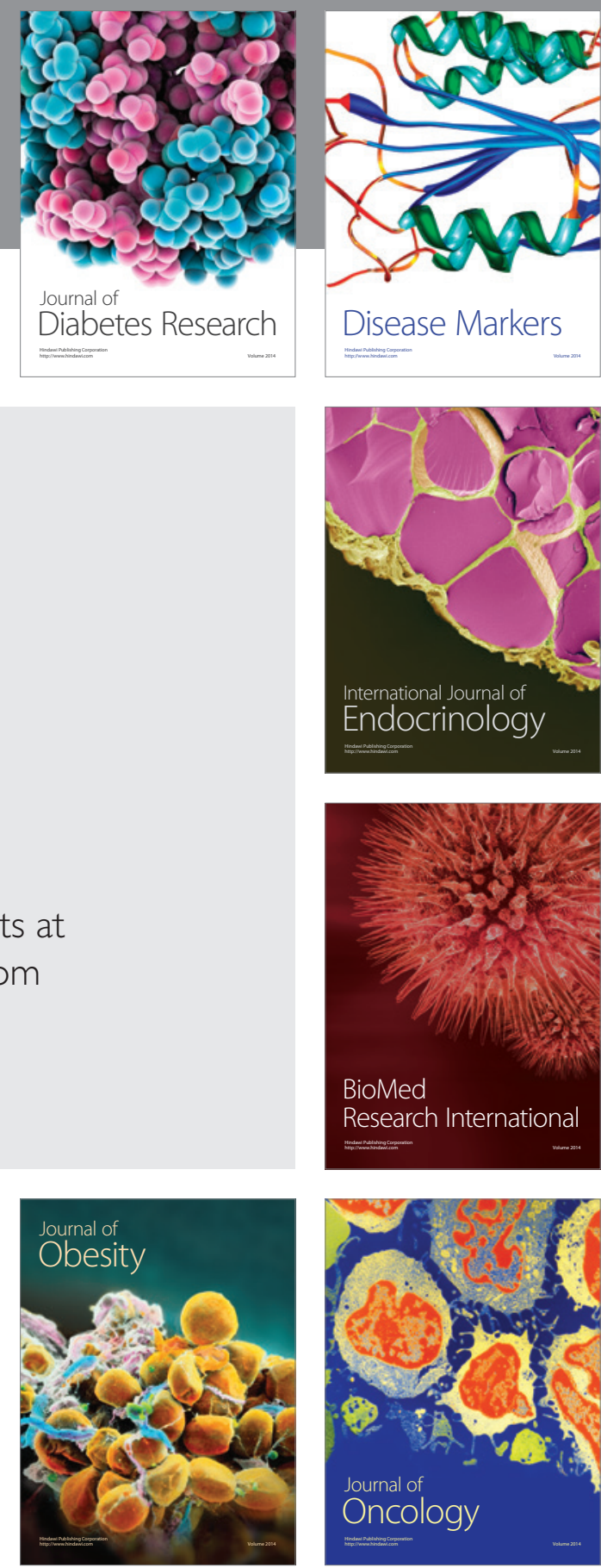

Disease Markers
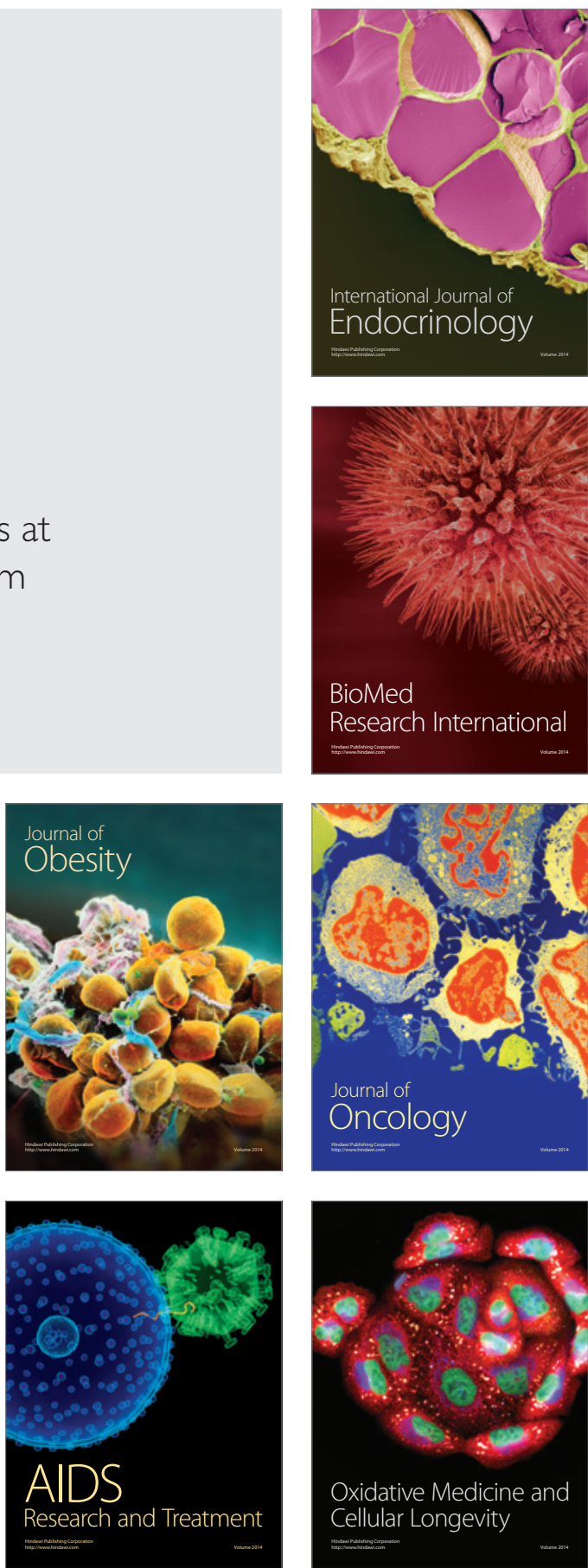